DrAFt VERSiOn June 11, 2018

Preprint typeset using $\mathrm{IAT}_{\mathrm{E}} \mathrm{X}$ style emulateapj v. 5/2/11

\title{
UNDERSTANDING BLACK HOLE MASS ASSEMBLY VIA ACCRETION AND MERGERS AT LATE TIMES IN COSMOLOGICAL SIMULATIONS
}

\author{
Andrea Kulier $^{1}$, Jeremiah P. Ostriker ${ }^{1,2}$, Priyamvada Natarajan ${ }^{3}$, Claire N. Lackner ${ }^{1,4}$, And Renyue Cen ${ }^{1}$ \\ Draft version June 11, 2018
}

\begin{abstract}
Accretion is thought to primarily contribute to the mass accumulation history of supermassive black holes throughout cosmic time. While this may be true at high redshifts, at lower redshifts and for the most massive black holes mergers themselves might add significantly to the mass budget. We explore this in two disparate environments - a massive cluster and a void region. We evolve SMBHs from $4>z>0$ using merger trees derived from hydrodynamical cosmological simulations of these two regions, scaled to the observed value of the stellar mass fraction to account for overcooling. Mass gains from gas accretion proportional to bulge growth and BH-BH mergers are tracked, as are black holes that remain "orbiting" due to insufficient dynamical friction in a merger remnant, as well as those that are ejected due to gravitational recoil. We find that gas accretion remains the dominant source of mass accumulation in almost all SMBHs; mergers contribute $2.5 \pm 0.1 \%$ for all SMBHs in the cluster and $1.0 \pm 0.1 \%$ in the void since $z=4$. However, mergers are significant for massive SMBHs. The fraction of mass accumulated from mergers for central black holes generally increases for larger values of the host bulge mass: in the void, the fraction is $2 \%$ at $M_{*, \text { bul }}=10^{10} M_{\odot}$, increasing to $4 \%$ at $M_{* \text {,bul }} \gtrsim 10^{11} M_{\odot}$, and in the cluster it is $4 \%$ at $M_{* \text {,bul }}=10^{10} M_{\odot}$ and $23 \%$ at $10^{12} M_{\odot}$. We also find that the total mass in orbiting SMBHs is negligible in the void, but significant in the cluster, in which a potentially detectable $40 \%$ of SMBHs and $\approx 8 \%$ of the total SMBH mas ${ }^{\circ}$ is found orbiting at $z=0$. The existence of orbiting and ejected SMBHs requires modification of the Soltan argument. We estimate this correction to the integrated accreted mass density of SMBHs to be in the range $6-21 \%$, with a mean value of $11 \pm 3 \%$. Quantifying the growth due to mergers at these late times, we calculate the total energy output and strain from gravitational waves emitted by merging SMBHs, and obtain a signal potentially detectable by pulsar timing arrays.
\end{abstract}

Subject headings: black hole physics — galaxies: nuclei — quasars: general

\section{INTRODUCTION}

Observations strongly suggest that supermassive black holes are harbored in the centers of almost all massive galaxies (Kormendy \& Richstone 1995). The masses of these central black holes are observed to correlate with multiple properties of their host galaxies. The most well-known and studied of these relations is the $M-\sigma$ relation between the mass of the black hole and the velocity dispersion of its host galaxy spheroid (Gebhardt et al. 2000; Ferrarese \& Merritt 2000; Tremaine et al. 2002; Gültekin et al. 2009). Relations between the black hole mass and the stellar mass and luminosity of the bulge have also been derived from observations (Marconi \& Hunt 2003; Häring \& Rix 2004; Beifiori et al. 2012; McConnell \& Ma 2013). It has also been claimed that the mass of the central black hole is correlated with galactic properties on scales larger than the bulge, such as the total stellar mass (Cisternas et al. 2011; Bennert et al. 2011; Beifiori et al.

\footnotetext{
akulier@princeton.edu

${ }^{1}$ Department of Astrophysical Sciences, Princeton University, Princeton, NJ 08544, USA

${ }^{2}$ Department of Astronomy, Columbia University, NYC, NY 10027, USA

${ }^{3}$ Department of Astronomy, 260 Whitney Avenue, Yale University, New Haven, CT 06511, USA

${ }^{4}$ Kavli Institute for the Physics and Mathematics of the Universe (WPI), Todai Institutes for Advanced Study, the University of Tokyo, Kashiwa, Japan

a where the total includes central, orbiting, and ejected SMBHs
}

2012) and luminosity (Läsker et al. 2014) of the host galaxy, and the mass of the entire host halo (Ferrarese 2002; Volonteri et al. 2011; see however Kormendy et al. 2011; Kormendy \& Bender 2011). There is also evidence of increased scatter in some of these relations for low mass galaxies (Greene et al. 2010b; Volonteri et al. 2011; McConnell \& Ma 2013). The existence of these correlations suggests interplay between black hole growth and star formation activity in galactic nuclei.

There appears to be a connection between AGN activity and star formation on galactic scales larger than the nucleus as well. The total AGN activity follows roughly the same trend as a function of cosmic redshift as the global star formation rate (Heckman et al. 2004; Merloni et al. 2004; Brusa et al. 2009; Shankar et al. $2009 \mathrm{~b})$. A correlation has also been found between the luminosity of individual AGN and the star formation luminosity of their host galaxies (Netzer 2009; Chen et al. 2013). Some have found that this correlation weakens or disappears for low-luminosity $\mathrm{AGN}$ and at $z \gtrsim 1$ (Rosario et al. 2012), but others claim that these observations can be explained if mean AGN activity follows host galaxy SFR on timescales $\gtrsim 100$ Myr but is highly variable on shorter timescales (Hickox et al. 2014). It is becoming clear at high redshifts that the global star formation rate tracking AGN activity does not imply that these processes occur in tandem in all individual galaxies (Treister et al. 2013).

While these relations imply that the evolution of a 
galaxy and its central black hole are intertwined, their exact origin remains uncertain. Several mechanisms have been proposed. One possibility is that the evolution of a galaxy regulates the growth of its central black hole by determining the amount of gas that finally reaches the black hole (Booth \& Schaye 2010, 2011). However, a SMBH can also regulate the evolution of its host galaxy through energy input via AGN feedback, which may be able to suppress star formation (Maiolino et al. 2012). Dubois et al. (2012) have run recent cosmological simulations including SMBH growth via accretion and mergers, as well as outflows and heating from AGN feedback, and conclude that AGN feedback is necessary to expel gas and suppress star formation. They also argue that AGN feedback is able to transform late-type galaxies into early-types and explain galaxy scaling relations such as the fundamental plane (Dubois et al. 2013). Similarly, Choi et al. (2014) simulate mergers of galaxies including radiative and mechanical AGN feedback and are able to recover the $M_{\bullet}-\sigma$ relation as well as reproduce the Xray luminosities of galactic halo gas seen in observations, although they find that AGN feedback is insufficient to explain galaxy size evolution.

It is not known which of the former two processes is dominant. A third explanation of the scaling relations is that some other factor — for instance, the total gas reservoir - regulates both the growth of the SMBH and the evolution of the galaxy. Bournaud et al. (2012) observed a correlation between giant clumps of gas and stars, which are indicative of violent disk instabilities, and AGN activity at $z \sim 0.7$. They propose that the evolution of disk instabilities, which cause gas inflow to the central bulge and SMBH, could produce the observed correlation between star formation and AGN activity (Bournaud et al. 2011).

Finally, a purely statistical explanation has been proposed for the existence of these observed correlations based on the idea that repeated mergers of galaxies, which also lead to the eventual mergers of their central black holes, can cause the SMBHs and their hosts to have correlated masses as a consequence of the central limit theorem (Hirschmann et al. 2010). One or a combination of the above mechanisms could be responsible for the observed scaling relations between SMBHs and their hosts. The argument based on the central limit theorem has recently been gaining strength as the evidence accumulates from both observations (van Dokkum et al. 2008) and theory (Oser et al. 2010) that the most massive galaxies grow their stellar components substantially via minor mergers during recent cosmic epochs. This is especially important for massive BCGs (brightest cluster galaxies) in rich clusters (Hausman \& Ostriker 1978; Lin et al. 2013). Evidence that BCGs have grown via mergers has been reinforced by a new statistical test (Lin et al. 2010). Recent observations of early-type galaxies showing that the color-magnitude and color-size relations change slope for $M_{*}>2 \times 10^{11}$, but the color- $\sigma$ relation stays constant, suggest that major dry mergers are significant in the evolution of such massive galaxies (Bernardi et al. 2011a,b). The impact of the increased frequency of mergers for the assembly of the stellar component suggests that they might play an important role in black hole growth as well at late times.

It is widely believed that black hole growth occurs primarily via accretion or merger triggered accretion episodes over cosmic time, although secular evolution driven by stellar evolutionary processes also appears to be important (Ciotti \& Ostriker 1997; Ostriker et al. 2010), especially for $z<2$. In massive galaxies, which have undergone multiple mergers, it is believed that most of the SMBH growth occurs in short accretion episodes fueled by gas flowing into the central region due to the merger or galactic cooling flows. SMBHs hosted in small galaxies that have not undergone any major mergers must, however, be supplied with gas through some different mechanism, such as dynamical relaxation and perhaps secular evolution processes (Sesana 2012). While these may indeed be the primary channel of growth during early times, it is becoming evident that the actual merger of black holes might contribute appreciably to the final mass inventory of the most massive black holes at low redshifts. This is suggested by the current evidence that minor mergers are a significant component of the late-time stellar mass growth of the most massive galaxies.

In this paper, we examine how gas inflows, mergers, and the large-scale environment determine black hole growth. To this end we track the growth histories of black holes in a typical over-dense cluster environment and an under-dense void environment. In particular, we know that the merger history in these environments at late times is divergent. Therefore, in this work we focus on quantifying the role that mergers play in the mass assembly history at late times. This has important consequences for the expected gravitational radiation from such events at low redshifts as well the observational implications for the number of wandering black holes. Since BH-BH mergers will be accompanied by the gravitational slingshot effect (Peres 1962; Bekenstein 1973; Fitchett \& Detweiler 1984), late time mergers may eject a significant amount of mass in BHs from merged galaxies. An inventory of BHs at the present epoch will, of course, not count this mass and thus will underestimate the $z=0$ cosmic mass density locked up in SMBHs. Furthermore, we expect that some SMBHs at any given time will be orbiting in the outskirts of the galactic potential - as the result of a lower velocity gravitational wave recoil or a recent merger of its host with a more massive galaxy. These orbiting $\mathrm{BHs}$ will also not be found in observations of galactic centers. The correction to the SMBH mass density from these two populations, which we estimate here, implies that the Soltan argument (Soltan 1982) will necessarily overestimate the efficiency of energy output from accreting BHs. This is because the energy output from accretion that is directly observed is incommensurate with the $\mathrm{SMBH}$ cosmic mass density estimated only from the census of $\mathrm{BHs}$ in galactic centers. Neglecting the "unmerged" SMBH population (i.e., ejected and orbiting $\mathrm{BHs}$ ) introduces errors in estimates of the inferred accretion efficiency for the population of BHs.

The outline of the paper is as follows: we first describe the key aspects of the problem that are tackled here - the role of late time mergers - in \$2. In \$3, the methodology and cosmological simulations used are detailed, followed by a discussion of previous work on this topic and our results in \$4. We conclude with a discussion of the implications and observational consequences 
of accounting for the role of mergers at late times on our current understanding of black hole growth.

\section{EXPLORING THE ROLE OF LOW-REDSHIFT MERGERS}

In this paper, we examine the consequences of mergers for the mass assembly history of SMBHs at late times and the observational consequences thereof. The "Soltan argument" (Soltan 1982) relates the observed luminosity density of AGN over time to the local mass density in SMBHs. The energy radiated by AGN is proportional to the rate at which they gain mass via accretion $L=\epsilon \dot{M}_{a c c} c^{2}$, where $\epsilon$ is the radiative efficiency factor. Since the total mass in SMBHs at $z=0$ is the integral of the mass they have accreted over all time, one can relate the observed luminosity density of AGN to the total mass density of SMBHs at $z=0$ (Shen 2009):

$$
\rho_{\bullet} \approx \int_{0}^{\infty} \frac{\mathrm{d} t}{\mathrm{~d} z} \mathrm{~d} z \int_{0}^{\infty} \frac{(1-\epsilon) L}{\epsilon c^{2}} \Phi(L, z) \mathrm{d} L
$$

where $\Phi(L, z)$ is the AGN bolometric luminosity function per $L$ interval. The local SMBH mass density $\rho_{\bullet}$ has been found to be consistent with the observed luminosity density of AGN if these AGN have a mean mass-to-energy conversion efficiency of $\epsilon \simeq 0.1$ (Yu \& Tremaine 2002; Marconi et al. 2004).

While SMBHs can grow through $\mathrm{BH}-\mathrm{BH}$ mergers as well as accretion, such mergers can only alter the shape of the SMBH mass function, having no significant effect on the total mass density of SMBHs (Menou \& Haiman 2004; Shen 2009; Shankar et al. 2009b). There is a small mass loss during mergers due to gravitational wave radiation, which is a negligible correction to the Soltan argument, but is important to know accurately for gravitational wave detection experiments (Rajagopal \& Romani 1995; Jaffe \& Backer 2003).

Many of the existing calculations of the energy emitted in gravitational waves during $\mathrm{BH}-\mathrm{BH}$ mergers use MonteCarlo dark matter merger trees (e.g., Wvithe \& Loeb 2003; Sesana et al. 2004; Enoki et al. 2004); however, these approaches do not take into careful account the enhanced merger rates in cluster environments where the most massive galaxies live (Volonteri \& Ciotti 2013). Some more recent works (Sesana et al. 2009; Kocsis \& Sesana 2011; Ravi et al. 2012) have used dark matter merger trees based on the Millennium Simulation (Springel et al. 2005). The dynamical influence of the galaxy stellar mass is not taken into account in dark matter-based merger trees, but it can greatly shorten the time for two galaxies to merge. Assuming an NFW density profile (Navarro et al. 1997) for the dark matter halo, the dynamical friction time for a galaxy in a subhalo to merge with the central galaxy has the form $t_{\mathrm{DF}} \propto\left(1+M_{*} / M_{\mathrm{DM}}\right)^{-9}$ (McWilliams et al. 2012), where $M_{*}$ is the stellar mass and $M_{\mathrm{DM}}$ is the halo mass of the tidally limited subhalo. Hydrodynamical cosmological simulations can account for the dynamics of both the dark and baryonic matter.

Other recent works have estimated the gravitational wave emission based on observational constraints. McWilliams et al. (2014) calculated the gravitational wave strain for $z \leq 1$ using observed galaxy mass functions combined with observed BH-galaxy scaling relations, assuming that SMBH growth at these times occurs purely via BH-BH mergers. Sesana (2013a, b) used observed galaxy stellar mass functions and pair fractions, combined with galaxy merger timescales from the Millennium Simulation and hydrodynamical simulations of galaxy mergers, to calculate galaxy merger rates. These rates were then combined with observed BH-galaxy scaling relations to obtain the expected strain.

Not all galactic mergers will result in black hole mergers, due to a finite dynamical friction time for the black hole to sink to the center of the galactic potential. There also exists the "final parsec problem" (see e.g., Milosavljević \& Merritt 2003), which is shorthand for the physical difficulties in bridging the gap between the binary separation reached by dynamical friction between the SMBH and the ambient stellar population and the much smaller separation at which gravitational radiation takes over as the primary angular momentum loss mechanism for the $\mathrm{BH}$ binary. We will not address this problem in this paper, but will assume that black holes effectively merge at galactic centers on the dynamical friction timescale.

The merger of two SMBHs can also impart a linear momentum to the resulting post-merger SMBH due to gravitational wave radiation (Peres 1962; Bekenstein 1973; Fitchett \& Detweiler 1984). This can cause the SMBH to be displaced from the center of the galaxy or ejected entirely; we discuss this in more detail in \$3.3.1.

Calculation of the mean mass-to-energy conversion efficiency of SMBHs using the Soltan argument requires an accurate census of the local mass density of SMBHs $\rho_{\bullet}$. Because we observe SMBHs only at the centers of galaxies, the existence of SMBHs outside of galactic centers due to the mechanisms described above implies that some of the local SMBH mass is unaccounted for by observations, and thus that the radiative efficiency $\epsilon$ derived from the Soltan argument is an overestimate. In this paper, we estimate the fraction of total SMBH mass that was accreted throughout cosmic time but is not currently observable in galactic centers, which must be added to the left side of Equation [1 in order to obtain the correct mean $\epsilon$. Unlike some semi-analytic works (Shankar et al. 2009b; Shen 2009; Shankar et al. 2013) that use observed AGN luminosity functions to constrain SMBH evolution, the merger trees we use in this work are entirely derived from hydrodynamical cosmological simulations and are thus independent of the assumed radiative efficiency.

Taking galaxy and black hole mergers as well as gravitational wave recoils explicitly into account will alter the observational consequences at low redshifts and that is precisely what we explore in detail in this paper. We use recent cosmological simulations (Cen 2011a b, 2012a, b, 2013) to study the relevance of different processes on the growth of SMBHs and their implications for the setting up of the various observed scaling relations in two significantly different environments. In our book-keeping we keep track of the total mass in black holes in the following four categories at redshifts between $z=4$ and 0 :

- The mass in central black holes acquired from accretion of gaseous matter.

- The mass in central black holes acquired from mergers with smaller black holes. 
- The mass in orbiting black holes that have not yet fallen to the center of the galactic potential.

- The mass in ejected black holes that have been kicked out due to gravitational radiation recoil.

The final two categories will be assessed as corrections to the normal Soltan-type arguments for estimating the revised efficiency of $\mathrm{BH}$-associated energy generation.

\section{METHODOLOGY AND DESCRIPTION OF COSMOLOGICAL SIMULATIONS}

As the basis for our analysis of SMBH evolution, we use the large-scale hydrodynamical galaxy simulations of Cen (2011a,b, 2012a,b, 2013). Detailed descriptions of the simulations can be found in the papers referenced above; we provide a brief overview in this section. We use galactic (not dark matter based) merger trees derived from these simulations as described in Lackner et al. (2012). The simulations are performed with the AMR (Adaptive Mesh Refinement) Eulerian hydrodynamics code Enzo (Bryan 1999; O'Shea et al. 2004; Joung et al. 2009). They consist of a low-resolution box of $120 \mathrm{~h}^{-1}$ $\mathrm{Mpc}$ on a side, and two high-resolution regions within this box, one containing a cluster with mass $\sim 3 \times 10^{14}$ $M_{\odot}$, and the other a void. These represent $+1.8 \sigma$ and $-1.0 \sigma$ fluctuations in the cosmic density field, respectively. These two extreme regions bracket the cosmic average environment. The cluster region box has dimensions $21 \times 24 \times 20 h^{-3} \mathrm{Mpc}^{3}$, and the void box has dimensions $31 \times 31 \times 35 h^{-3} \mathrm{Mpc}^{3}$. The dark matter particle mass is $1.07 \times 10^{8} h^{-1} M_{\odot}$, while the stellar particle mass is generally around $10^{6} M_{\odot}$. The resolution in the cluster and void regions is always better than $460 h^{-1}$ pc physical. Galaxies in the simulation box are identified by using the HOP algorithm on stellar particles (Eisenstein \& Hu 1999).

The simulation includes prescriptions for UV background (Haardt \& Madau 1996), shielding from UV radiation by neutral hydrogen (Cen et al.|2005), metallicitydependent radiative cooling (Cen et al.|1995), formation of star particles from gas (Cen \& Ostriker 1992), and supernovae feedback (Cen et al. 2005). It does not include feedback from AGN, which may be partly the reason that the largest galaxies in the simulation box have too many stars in comparison with observed relations. This is a well known problem in hydrodynamical simulations (Oser et al. 2010; Guo et al. 2010) and one that does exist in our version. Broad agreement is otherwise found between the simulation results and observations (Cen 2011a).

The simulations use the following cosmological parameters, consistent with Komatsu et al. (2009): $\Omega_{M}=$ $0.28, \Omega_{b}=0.046, \Omega_{\Lambda}=0.72, \sigma_{8}=0.82, H_{0}=100 h^{-1}$ $\mathrm{Mpc}^{-1}=70 \mathrm{~km} \mathrm{~s}^{-1} \mathrm{Mpc}^{-1}$, and $n=0.96$. These are also the values we adopt throughout this paper in our calculations.

A merger tree is created from this simulation. In the cluster box, there are 38 redshift slices from $z=4$ to $z=0$ with $\Delta z=0.05$ for $z<1.35$, and slices at $z=1.5$, $1.6,1.75,1.9,2.0,2.2,2.5,2.8,3.1$, and 4 . In the void box, there are 14 redshift slices between $z=0$ and $z=4$, at $z=0,0.05,0.15,0.2,0.4,0.5,0.6,0.8,1.0,1.6,1.9$, 2.5, 3.1 and 4 (Lackner et al. 2012).

Since our focus is on massive galaxies, and due to the resolution limit of the simulation, our merger tree contains only galaxies with stellar masses $M_{*}>10^{9} M_{\odot}$. In the void box, the grouping algorithm identifies some groupings of particles as galaxies that do not exist in later redshift slices. For our purposes, we ignore any groupings of particles that do not have a descendant at $z=0$.

We first scale down the stellar masses of the galaxies in this merger tree to account for the overproduction of stellar mass in our simulation. We then seed these galaxies with central black holes whose evolution we trace based on the evolution of their host galaxies. Further details are provided below.

\subsection{Scaling of Cluster and Void Boxes}

Our simulation considerably overproduces stellar mass compared to the amount of dark matter present. This is a common problem in cosmological simulations (Oser et al. 2010; Guo et al. 2010). Because observations find that the masses of central black holes scale with the stellar content of their host galaxies, using the galaxy masses as-is would lead us to greatly overestimate the amount of mass contained in black holes. We attempt to allow for this by scaling down the stellar masses of our galaxies by a constant factor in proportion to the excess of star formation efficiency as normalized by current observations.

In the cluster, the stellar mass is $3 \times 10^{13} M_{\odot}$ within $r_{200}$, the radius within which the mean density is equal to 200 times the critical density. The dark matter mass within this same radius is $3 \times 10^{14} M_{\odot}$ (Lackner et al. 2012). This implies a stellar to dark matter ratio of 0.1 within the virial radius. Comparing this to the stellar-halo mass relation found by Leauthaud et al. (2012) using weak lensing and halo occupation distribution methods, combined with the fraction of halo mass that is in gas given by Pratt et al. (2009), one finds that our simulation overproduces stars for a cluster of this mass by a factor of roughly 4 to 6 . Determinations of the stellar-halo mass relation found by matching simulated dark matter halos to observed galaxy mass functions (Guo et al. 2010; Behroozi et al. 2010; Moster et al. 2013) find somewhat lower values than Leauthaud et al. (2012), implying an even larger excess for our simulation. Recent observational determinations of low redshift cluster stellar masses using WISE and 2MASS and halo masses using Chandra find values of the stellar to dark matter ratio in the range $\approx 0.01-0.03$ (Lin et al. 2012) within $r_{500}$. This corresponds to an overproduction of stellar mass by a factor of approximately 3 to 10 . Given these results, we assume the stellar to dark matter mass ratio within the virial radius of the cluster to be 0.02 , implying a scaling factor for galaxies in the cluster of 0.2 . We apply this scaling factor to all the galaxies in the cluster box (not only those within the virial radius), within which the initial stellar to dark matter mass ratio is 0.06 . Thus, after scaling, this ratio is 0.012 for the entire cluster box. However, because overcooling is less of a problem in the outskirts of the cluster region than the center, this stellar to dark matter mass ratio may be somewhat too low. 
Due to the lack of observational data for the star formation efficiency specifically in the void, we assume arbitrarily that the stellar to dark matter mass ratio in the void is half of the mean ratio of the universe. We calculate the mean universal value using the $z=0$ stellar mass density obtained by Bernardi et al. (2013) for a Sersic + Exponential fit to galaxy luminosity profiles: $\rho_{*}=3.30 \times 10^{8} M_{\odot} \mathrm{Mpc}^{-3}$. Although this value is for galaxies with $M_{*}>10^{9} M_{\odot}$, galaxies with lower stellar masses are expected to contribute a negligible amount of the total stellar mass in the universe (Brinchmann et al. 2004). This results in a mean stellar to dark matter mass ratio of 0.0104 , and thus a ratio of 0.0052 for the void. The stellar to dark matter ratio for the entire void box in our simulation is 0.012 (Lackner et al. 2012), so we scale the stellar masses of the galaxies in the void by a factor of 0.43 in the same manner that we do for the cluster.

It should be noted that these scalings for the void and cluster box are simple and do not reflect the more complex trends in stellar mass overproduction in our simulation. A discussion of the effect of our choices for the scaling can be found in $\$ 4.2$.

When evolving our SMBHs, we grow them proportionally to the bulge mass in their host galaxy (see $\$ 3.2$ below for more detail), in agreement with observations. We set the proportionality constant so that the resulting SMBH mass per unit dark matter matches that observed in cluster and void regions in the real universe. To accomplish this, we first estimate the fraction of stellar mass in bulges in cluster and void regions. We use a mean bulge-to-total mass ratio as function of stellar mass of

$$
\frac{M_{*, \text { bulge }}}{M_{*}}=\left[1+\left(\frac{M_{*}}{2 \times 10^{10} M_{\odot}}\right)^{-0.58}\right]^{-1} .
$$

This is consistent with the bulge mass fractions obtained from a sample of $\sim 660,000$ SDSS galaxies in Mendel et al. (2014) (aside from a drop in bulge fraction at $M_{*} \geq 10^{11} M_{\odot}$ not seen in previous studies, e.g. Gadotti 2009; Cibinel et al. 2013). We obtain galaxy stellar mass functions for high and low density regions of the universe from Bolzonella et al. (2010). Combining these with the bulge-to-total mass ratio as a function of stellar mass results in a bulge stellar mass fraction of $71 \%$ in the cluster and $51 \%$ in the void. We thus find a bulge stellar mass to dark matter mass ratio of $8.52 \times 10^{-3}$ in the cluster and $2.65 \times 10^{-3}$ in the void. We note that we normalize the entire cluster box as though it had the stellar mass function of a high density region, which may not be the case for the outskirts of the box. Thus, while the stellar to dark matter ratio in the cluster box is likely somewhat underestimated, the fraction of stellar mass in bulges is likely somewhat overestimated.

Using these values for the bulge mass per unit dark matter, we derive the SMBH mass per unit dark matter. We use the observed $M_{\bullet}-M_{\text {bulge }}$ relation from McConnell \& Ma (2013), which implies a mean black hole to bulge mass ratio of $\approx 4 \times 10^{-3}$ when taking into account the scatter in the relation. We assume this value for both the cluster and void regions and obtain a SMBH mass per unit dark matter mass of $3.41 \times 10^{-5}$ in the cluster and $1.06 \times 10^{-5}$ in the void. We grow the SMBHs in proportion to their host bulges with a distribution such that we obtain these values at $z=0$ in the cluster and void boxes; a full description of the SMBH growth prescriptions is given in $\$ 3.2$

The cluster and void boxes in the cosmological simulation we use are $+1.8 \sigma$ and $-1 \sigma$ fluctuations in the cosmic density field, respectively, and were chosen so as to bracket the "global average" of various physical quantities (Cen 2011a). To approximate this global average, we combine the rescaled quantities from the void and cluster boxes in a weighted average. We choose weights such that the fraction of stellar mass per unit dark matter mass matches $\rho_{*} / \rho_{\text {DM }}$ in the local universe at $z=0$. We use the local stellar mass density of $3.3 \times 10^{8} M_{\odot} \mathrm{Mpc}^{-3}$ for galaxies with $M_{*}>10^{9} M_{\odot}$ found in Bernardi et al. (2013), resulting in a stellar to dark matter mass ratio of 0.0104 . We note that the value of the local stellar mass density used also carries significant uncertainty, and depends on both the assumed initial mass function and stellar mass-to-light ratio of galaxies, as well as the photometry used to obtain the galaxy luminosity functions from which the stellar mass functions are derived (Bernardi et al. 2013). To match this stellar to dark matter mass ratio at $z=0$ requires that we approximate the universe as $77 \%$ cluster per unit dark matter mass and $23 \%$ void. We use these same weights at all redshifts.

We multiply by the dark matter density of the universe where necessary to obtain various number and mass densities cited throughout our paper. The black hole to dark matter mass ratios derived above for the universe correspond to a universal SMBH mass density of $9.1 \times 10^{5} M_{\odot} / \mathrm{Mpc}^{-3}$. This value is higher than previous estimates, which are in the range $3-5.5 \times 10^{5} \mathrm{M}_{\odot} /$ $\mathrm{Mpc}^{-3}$ (Shankar et al. 2009b). The main source of the difference is the $\mathrm{BH}$ to bulge mass ratio implied by the observations of McConnell \& Ma (2013), which is higher than that used to obtain previous estimates of the SMBH mass density (see also Kormendy \& Ho 2013).

We run multiple realizations of our randomized model and use them to compute one-sigma errors on the results we obtain. However, it should be noted that although our black hole growth is modeled with random scatter, we are always using the same galaxy merger trees obtained from the simulations of Cen (2011a).

\subsection{Black Hole Evolution Prescriptions}

The masses of central supermassive black holes are known to correlate well with the masses of host elliptical galaxies and the bulge component of host spirals. Correlation with the disk of spiral galaxies seems to be weaker or nonexistent (e.g., Kormendy \& Ho 2013 and references therein; see however Läsker et al. 2014). Thus we take the growth of our SMBHs to be proportional to that of the bulge stellar mass in our galaxies. However, our simulation does not have the resolution to distinguish bulges from disks, so we assume that the bulge mass of galaxies is a function of their stellar mass following Equation 2 .

For simplicity we assume the same relation between bulge and total stellar mass at all redshifts, although it is expected from models that the bulge mass fraction at fixed stellar mass should increase with decreasing redshift (Somerville et al.|2012; Guo et al.|2013; Avila-Reese et al. 2014). Recent observations also show 
an increase in bulge stellar mass fraction between $z=2.5$ and $z=0.5$, although the increase is slight (Lang et al. 2014). Since we do not take such evolution into account, it is likely that a larger fraction of SMBH growth takes place at early times in our model than in the real universe. We grow our SMBHs such that the black hole mass to dark matter mass ratio at $z=0$ is correct by design (\$3.1), meaning that the SMBHs we obtain at early times may be overly massive.

We place a seed black hole in any galaxy that reaches our lower mass limit at any redshift; this is $M_{*}=10^{9} M_{\odot}$ for the original simulation masses, and thus $2 \times 10^{8} M_{\odot}$ for the cluster and $4.3 \times 10^{8} M_{\odot}$ for the void in terms of rescaled masses. The seed black hole is taken to have

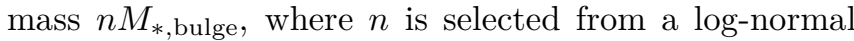
distribution with median value such that the black hole to dark matter mass ratio at $z=0$ matches observations (\$3.1), and with scatter as described further below. The median proportionality factor is $1.50 \times 10^{-3}$ for the cluster and $1.25 \times 10^{-3}$ for the void. This is consistent with the common assumption that of the gas added to (and retained by) galaxies, approximately one part in a thousand is accreted onto the central black hole, with most of the remainder transformed into stars (Li et al. 2007). We note that this assignment of $\mathrm{BH}$ seed masses as early as $z=4$ pre-supposes the existence of a scaling relation at this epoch akin to what is empirically measured at $z=0$.

SMBHs are then allowed to grow through BH-BH mergers and accretion of gaseous material from the galaxy. We calculate the accreted and merged mass by considering all parent black holes of a single black hole to be "merged mass" except for the most massive one. We adopt a simple prescription for accretion in which the mass accreted by the central black hole in each redshift slice is proportional to the stellar mass formed in its host galaxy in that redshift slice times the bulge fraction of the host galaxy, with some scatter in the assumed proportionality factor. A physical mechanism proposed for such a proportionality is that gas infall onto the $\mathrm{SMBH}$ is caused by the gas drag due to stellar radiation, which in turn is roughly proportional to the star formation rate (Umemura 2001; Kawakatu \& Umemura 2002; Kawakatu et al. 2003; Granato et al. 2004). Because stars are sometimes ejected from galaxies in the simulation, it is possible for the total stellar mass of a galaxy to occasionally decrease. If this is the case, we assume zero accretion onto the black hole in that redshift slice. Otherwise, the mass accreted by the black hole is

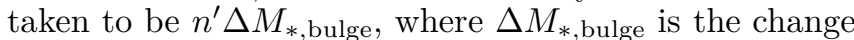
in bulge stellar mass due to star formation in that redshift slice, and $n^{\prime}$ is a proportionality factor chosen from the same log-normal distribution as the proportionality factor for the seeding prescription. We choose such a prescription for accretion due to the observed correlation between AGN activity and galactic star formation rate (Heckman et al. 2004; Merloni et al. 2004; Brusa et al. 2009; Shankar et al. 2009b).

The proportionality factors for the seed masses and $\mathrm{BH}$ accretion rates are chosen from the same log-normal distribution, with median values $1.50 \times 10^{-3}$ for the cluster and $1.25 \times 10^{-3}$ for the void as described above. We adjust the scatter of the distribution so as to produce an intrinsic scatter of $\sim 0.35$ in the cluster $M_{\bullet}-M_{* \text {, bulge }}$ relation at $z=0$, similar to observed values reported in the literature (Sani et al. 2011; McConnell \& Ma 2013).

When a smaller galaxy merges into a larger one, we calculate the dynamical time for the black hole from the smaller galaxy to move to the center of the newly formed merged galaxy. We calculate the dynamical friction time as:

$$
t_{\mathrm{DF}}=\frac{19 \mathrm{Gyr}}{\ln \left(1+M_{*} / M_{\bullet}\right)}\left(\frac{R_{e}}{5 \mathrm{kpc}}\right)^{2} \frac{\sigma}{200 \mathrm{~km} / \mathrm{s}} \frac{10^{8} M_{\odot}}{M_{\bullet}} f_{e} .
$$

The equation is that for a circular orbit from Binney \& Tremaine (1987) and is corrected by a factor $f_{e}$ for the ellipticity of the orbit. Following the analysis of Gnedin et al. (2014), who find that $f_{e} \simeq 0.39-0.76$ for satellite halos merging with a central galaxy, we adopt $f_{e}=0.5$. Although choosing a different value of $f_{e}$ will obviously alter the number of orbiting BHs and the number of BH-BH mergers by increasing or decreasing the dynamical friction time, the difference is not large enough to significantly affect our general results. For example, adopting even the extreme case of purely circular orbits $\left(f_{e}=1\right)$ increases the fraction of mass in orbiting BHs at $z=0$ from $7 \%$ to $10 \%$, while reducing the mass contribution from $\mathrm{BH}-\mathrm{BH}$ mergers in massive $\mathrm{BHs}$ from $\approx 24 \%$ to $\approx 20 \%$.

We obtain values for the effective radius $R_{e}$ and velocity dispersion $\sigma$ of our simulated galaxies by using observed fits to the stellar mass from SDSS data at $z=0$ (Nipoti et al. 2009). Since the relations between galactic mass, radius, and velocity dispersion are known to evolve with redshift, we take $R_{e} \propto(1+z)^{-0.98}$ at fixed mass based on the observations compiled in van der Wel et al. (2008) and McLure et al. (2013). Similarly, we take $\sigma \propto(1+z)^{0.47}$ based on observations of the mass fundamental plane out to $z \sim 2$ (Bezanson et al. 2013). Also, both the size and velocity dispersion evolution are consistent with the recent observations of Belli et al. (2014) for $0.9<z<1.6$. Thus our final scaling relations are:

$$
\begin{aligned}
R_{e} & =2.5 \mathrm{kpc}\left(\frac{M_{*}}{10^{11} M_{\odot}}\right)^{0.73}(1+z)^{-0.98}, \\
\sigma\left(R_{e}\right) & =190 \mathrm{~km} / \mathrm{s}\left(\frac{M_{*}}{10^{11} M_{\odot}}\right)^{0.2}(1+z)^{0.47} .
\end{aligned}
$$

If a galaxy is involved in another merger before a satellite black hole has merged with the central one, we recalculate the dynamical friction time. If the satellite black hole is in the more massive galaxy, we take the new dynamical friction time to be the smaller of the remaining time to black hole merger and the dynamical friction time that would be calculated for the post-merger galaxy. For a less massive galaxy merging into a more massive one, its satellite and central black holes are taken to have a dynamical friction time calculated for the new post-merger galaxy.

If a black hole is ejected or displaced from the center of a galaxy due to a gravitational wave recoil (see \$3.3.1 below), the galaxy may have no central SMBH. If a galaxy lacking a central $\mathrm{BH}$ produces an additional $10^{9} M_{\odot}$ in unscaled galactic star formation (i.e., $2 \times 10^{8} M_{\odot}$ in the cluster and $4.3 \times 10^{8} M_{\odot}$ in the void after scaling), we seed 
it again with a black hole with mass proportional to the (scaled) mass in stars formed times the bulge mass fraction, where the proportionality constant is chosen from the same log-normal distribution as for the seeds and accreted mass described earlier.

\subsection{Low redshift merging and gravitational radiation}

We calculate the gravitational radiation luminosity expected based on the $\mathrm{BH}-\mathrm{BH}$ mergers that occur in our simulation. This energy is equivalent to the mass lost from the black hole pair as they are merging. For each $\mathrm{BH}-\mathrm{BH}$ merger, we apply an approximation for the energy emitted in gravitational waves from Barausse et al. (2012):

$$
\begin{aligned}
\frac{E_{\text {rad }}}{M}= & {\left[1-\tilde{E}_{\mathrm{ISCO}}(\tilde{a})\right] \nu+} \\
& 4 \nu^{2}\left[4 p_{0}+16 p_{1} \tilde{a}(\tilde{a}+1)+\tilde{E}_{\mathrm{ISCO}}(\tilde{a}-1)\right] .
\end{aligned}
$$

Here $M \equiv m_{1}+m_{2}$ is the total mass of the two black holes and $\nu \equiv m_{1} m_{2} / M^{2}$ is the symmetric mass ratio. The constants $p_{0}$ and $p_{1}$ come from a polynomial fit to the emitted energy, and have the values:

$$
p_{0}=0.04827 \pm 0.00039, \quad p_{1}=0.01707 \pm 0.00032 .
$$

Here $\tilde{E}_{\mathrm{ISCO}}$ is the energy per unit mass at the innermost stable circular orbit and is given by:

$$
\tilde{E}_{\mathrm{ISCO}}(\tilde{a})=\sqrt{1-\frac{2}{3 \tilde{r}_{\mathrm{ISCO}}^{e q}}(\tilde{a})},
$$

where

$$
\begin{aligned}
& \tilde{r}_{\mathrm{ISCO}}^{e q}(\tilde{a})=3+Z_{2} \\
& \quad-\operatorname{sign}(\tilde{a}) \sqrt{\left(3-Z_{1}\right)\left(3+Z_{1}+2 Z_{2}\right)}, \\
& Z_{1}=1+\left(1-\tilde{a}^{2}\right)^{1 / 3}\left[(1+\tilde{a})^{1 / 3}+(1-\tilde{a})^{1 / 3}\right], \\
& Z_{2}=\sqrt{3 \tilde{a}^{2}+Z_{1}^{2}} .
\end{aligned}
$$

In all the above equations, $\tilde{a}$ is defined as

$$
\tilde{a} \equiv \frac{\hat{\boldsymbol{L}} \cdot\left(\boldsymbol{S}_{1}+\boldsymbol{S}_{2}\right)}{M^{2}}=\frac{\left|\boldsymbol{a}_{1}\right| \cos \beta+q^{2}\left|\boldsymbol{a}_{2}\right| \cos \gamma}{(1+q)^{2}},
$$

where $q \equiv m_{1} / m_{2}<1$ is the mass ratio of the two black holes, $\left|\boldsymbol{a}_{1}\right|$ and $\left|\boldsymbol{a}_{2}\right|$ are the spin magnitudes, and $\beta$ and $\gamma$ are the angles between the orbital angular momentum unit vector $\hat{\boldsymbol{L}}$ and the spins of the first and second black hole, respectively. By construction, this formula is accurate in both the test-particle limit and for equal-mass binaries.

A number of works have investigated the expected distributions of the magnitude and orientation of $\mathrm{SMBH}$ spin vectors, using both semi-analytic models (e.g., Volonteri et al. 2005; Barausse 2012; Volonteri et al. 2013) and hydrodynamical simulations (e.g., Dotti et al. 2013; Dubois et al. 2014). SMBHs are spun up by coherent gas accretion and spun down by chaotic accretion (Volonteri et al. 2013; Barausse 2012). In a gaspoor merger, the spin directions of the merging $\mathrm{BHs}$ are expected to be randomly distributed (Bogdanović et al. 2007; Barausse 2012), and the merger remnant tends to be spun down compared to the initial central $\mathrm{BH}$ (Volonteri et al. 2013). Alternatively, in a gas-rich merger, gas accretion can exert torques that align the spins of both BHs with the angular momentum of the gas accretion disk to within $10^{\circ}-30^{\circ}$ (Bogdanović et al. 2007; Dotti et al. 2010), which creates a spun-up merger remnant with the same spin direction as the two inspiraling BHs (Dotti et al. 2010). However, quantitative predictions of trends in black hole spin with SMBH mass and redshift tend to vary between models and depend on the assumptions made. For the main results presented in this paper, we make the most "simple" possible assumption by selecting spin parameter values from a random uniform distribution between 0 and 1 , and taking the direction of the spins to be uniformly randomly oriented over a sphere. This is similar to the assumptions made by Schnittman \& Buonanno (2007) when calculating the expected recoil velocity distribution of SMBHs. If we assume the opposite extreme - that all merging BHs are aligned with the orbital angular momentum to within $10^{\circ}$ - the only result that is significantly affected is the number of ejected BHs. We describe the effect of assuming partially aligned $\mathrm{BH}$ spins in \$4.2.

\subsubsection{Gravitational Wave Recoils}

When two orbiting supermassive black holes merge, the gravitational radiation produced can impart a linear ("slingshot") momentum to the SMBH resulting from the merger (Peres 1962; Bekenstein 1973; Fitchett \& Detweiler 1984). Recent numerical simulations of general relativity show that such "kicks" can in some cases be large enough to exceed the escape velocity of the host galaxy and eject the resulting SMBH (Herrmann et al. 2007; Koppitz et al. 2007; Campanelli et al. 2007a,b; Lousto \& Zlochower 2011). Gravitational wave recoils with velocities insufficient to eject the SMBH can still displace it from the center of the galaxy, to which it may return via dynamical friction.

We account for the effects of gravitational wave "kicks" on our population of SMBHs, including both ejections and displacements from the center of the galaxy. We use the fitting formula from Lousto et al. (2012) for the velocity imparted to an $\mathrm{SMBH}$ resulting from a merger:

$$
\begin{aligned}
& \boldsymbol{V}_{\text {recoil }}(q, \boldsymbol{\alpha})=v_{m} \hat{\boldsymbol{e}}_{1}+v_{\perp}\left(\cos \xi \hat{\boldsymbol{e}}_{1}+\sin \xi \hat{\boldsymbol{e}}_{2}\right)+v_{\|} \hat{\boldsymbol{n}}_{\|}, \\
& v_{m}=A_{m} \frac{\eta^{2}(1-q)}{(1+q)}\left[1+B_{m} \eta\right] \\
& v_{\perp}=H \frac{\eta^{2}}{(1+q)}\left[\left(\alpha_{2}^{\|}-q \alpha_{1}^{\|}\right)\right] \\
& v_{\|}=16 \eta^{2} /(1+q)\left[V_{1,1}+V_{A} \tilde{S}_{\|}+V_{B} \tilde{S}_{\|}^{2}+V_{C} \tilde{S}_{\|}^{3}\right] \\
& \times\left|\boldsymbol{\alpha}_{2}^{\perp}-q \boldsymbol{\alpha}_{1}^{\perp}\right| \cos \left(\phi_{\Delta}-\phi_{1}\right) .
\end{aligned}
$$

Here $\eta=q /(1+q)^{2}$, where $q=m_{1} / m_{2}$ is the mass ratio of the smaller to larger black hole, $\boldsymbol{\alpha}_{i}=\boldsymbol{S}_{i} / m_{i}^{2}$ is the dimensionless spin of black hole $i$, $\|$ and $\perp$ refer to components parallel and perpendicular to the orbital angular momentum, respectively, $\hat{e}_{1}$ and $\hat{e}_{2}$ are orthogonal unit vectors in the orbital plane, $\xi$ is the angle between the unequal mass and spin contributions to the recoil velocity in the orbital plane, and $\tilde{\boldsymbol{S}}=2\left(\boldsymbol{\alpha}_{2}+q^{2} \boldsymbol{\alpha}_{1}\right) /(1+q)^{2}$. $\phi_{\Delta}$ is the angle between the in-plane component $\boldsymbol{\Delta}^{\perp}=$ 
$\left(m_{1}+m_{2}\right)\left(\boldsymbol{S}_{2}^{\perp} / m_{2}-\boldsymbol{S}_{1}^{\perp} / m_{1}\right)$ and the infall direction at merger. The coefficients are obtained numerically and are $H=6.9 \times 10^{3}, A_{m}=1.2 \times 10^{4}, B_{m}=-0.93$, $V_{1,1}=3677.76 \mathrm{~km} / \mathrm{s}, V_{A}=2481.21 \mathrm{~km} / \mathrm{s}, V_{B}=1792.45$ $\mathrm{km} / \mathrm{s}$, and $V_{C}=1506.52 \mathrm{~km} / \mathrm{s}$ (Lousto et al. 2012). This fitting formula is similar to those obtained by previous authors, e.g. Campanelli et al. (2007a); Baker et al. (2008); van Meter et al. (2010). We adopt a value of $\xi=145^{\circ}$ based on the numerical results for quasi-circular merger configurations from Lousto \& Zlochower (2008). The angle $\phi_{1}$ depends on the mass ratio and initial separation of the merging $\mathrm{BHs}$, and can only be computed in numerical relativistic simulations; since we are only interested in the statistical distribution of kick velocities, we take $\phi_{1}=0$ and define $\phi_{\Delta}$ with respect to a fixed arbitrary direction, as recommended in Lousto et al. (2010).

We assume randomly distributed spin magnitudes and spin directions with respect to the orbital angular momentum as described in $\$ 3.3$. The recoil velocity is highly sensitive to the orientation of the spins relative to the angular momentum.

To calculate the trajectory of the kicked SMBH, we follow a similar prescription as Madau \& Quataert (2004): we assume the density profile of the galaxy is a truncated isothermal sphere, with a core radius equal to the radius of gravitational influence of the post-merger SMBH, $R_{\mathrm{BH}} \approx G M_{\mathrm{BH}} / \sigma^{2}$, so that $\rho(r)=\sigma^{2} /\left[2 \pi G\left(r^{2}+R_{\mathrm{BH}}^{2}\right)\right]$. The velocity dispersion $\sigma$ is obtained from Equation 5 . If the $\left|\boldsymbol{V}_{\text {recoil }}\right|$ from gravitational wave radiation is found to be larger than the isothermal sphere escape speed $2 \sigma\left(\ln \left(R_{e} / R_{\mathrm{BH}}\right)\right)^{1 / 2}$, where the effective radius $R_{e}$ is obtained from Equation 4, we assume the black hole is ejected from the galaxy. If the kick is insufficient to eject the black hole, we calculate the time for the displaced SMBH to return to the center of the galaxy via dynamical friction.

We approximate the orbits of the kicked BHs as purely radial. We solve numerically for the radial position of the $\mathrm{SMBH}$ as a function of time on a radial orbit using the equation of dynamical friction

$$
\begin{aligned}
\frac{d^{2} \boldsymbol{r}}{d t^{2}} & =-\frac{G M(r)}{r^{2}} \hat{\boldsymbol{r}} \\
& -\frac{4 \pi G^{2} \rho M_{\text {cusp }} \ln \Lambda}{v^{2}}\left(\operatorname{erf}(x)-\frac{2 x}{\sqrt{\pi}} e^{-x^{2}}\right) \hat{\boldsymbol{v}},
\end{aligned}
$$

where $M(r)$ is the mass within radius $r, x=v / \sqrt{2} \sigma$, and the Coulomb logarithm $\ln \Lambda$ is taken to be equal to 1, for reasons described in Madau \& Quataert (2004) and Maoz (1993). We take $M_{\text {cusp }}=2 M_{\mathrm{BH}}$ for $V_{\text {recoil }}<\sigma$ and $M_{\text {cusp }}=M_{\mathrm{BH}}$ otherwise. It should be noted that the dynamical friction times for radial orbits are highly dependent on the assumed central density (or equivalently, core radius), and so are only a rough approximation.

As described in Madau \& Quataert (2004), a kicked $\mathrm{BH}$ would likely gain some angular momentum traveling through the galaxy so that its orbit was no longer purely radial, which would lengthen the time for it to return to the center. To test the impact of this, we also calculated the dynamical friction time of all recoiling $\mathrm{BHs}$ using Equation 3 with $f_{e}=0.5$ and an initial radius of $\left.R_{\mathrm{BH}} \exp \left[\left(V_{\text {recoil }} / 2 \sigma\right)^{2}\right)\right] / \sqrt{e}$, and found the effect on our results to be negligible.

\section{DISCUSSION}

\subsection{Previous work}

The evolution of supermassive black holes through cosmic time has been modeled using different approaches to tracking the dark matter halos they reside in, including Monte Carlo merger trees (e.g., Haehnelt \& Kauffmann 2000; Lippai et al. 2009; Nataraian 2011), PressSchechter theory (e.g., Yoo et al. 2007), and cosmological simulations (e.g., Di Matteo et al. 2003; Sijacki et al. 2009). A more complete review of the early models of the growth of SMBHs is given in Natarajan (2004).

Some works have focused purely on the feedback between the AGN and its galaxy without consideration of mergers. One example is the semi-analytic model of Granato et al. (2004). In this model, star formation causes accretion onto the central black hole via radiation drag; the black hole consequently emits radiation and kinetic outflows which expel gas and quench star formation. Star formation also results in supernovae, which similarly expel the gas. The feedback from supernovae and AGN results in galaxy downsizing. The galaxy first goes through a phase as a dust-shrouded submillimeter galaxy (SMG) involving rapid star formation and accretion onto the black hole, which then induces feedback that causes the galaxy to become red and early-type. The model has been able to match observed properties of galaxies, such as the masses and accretion rates of SMGs (Granato et al. 2006), and the local early-type galaxy scaling relations (Cirasuolo et al. 2005) as well as their size evolution (Fan et al. 2008). It is also able to reproduce the mass functions (Granato et al. 2004) and hard X-ray and optical luminosity functions (Lapi et al. 2006) of quasars.

Most studies have focused on understanding black hole growth in individual galaxies residing in average overdensity environments, typically the field. There have been few studies of black hole mass assembly in extremely over-dense cluster and under-dense void environments. Using dark matter merger trees derived from Press-Schechter theory, Yoo et al. (2007) examined the expected growth of black holes due to mergers in a large cluster with halo mass $10^{15} h^{-1} M_{\odot}$ at late times. They found that most SMBHs in the cluster with masses $\gtrsim 10^{7.5} M_{\odot}$ underwent mergers, with the most massive SMBHs increasing their mass by approximately a factor of 2 since $z=2$. Although the central galaxy generally contained the most massive SMBH at $z=0$, Yoo et al. (2007) found that for some cluster assembly histories, the most massive SMBH may be hosted by a satellite galaxy.

Current simulations do not agree on the evolution of BH-galaxy scaling relations with redshift. Some find that SMBHs at higher redshift should be more massive than if they followed the $z=0 M_{\bullet}-\sigma$ relation (Hopkins et al. 2009; Dubois et al. 2012), while others find that they should be less massive (Malbon et al. 2007; Di Matteo et al. 2008). Some observational studies of broad line AGN samples at redshifts up to $z \sim 4$ find that these SMBHs are more massive at fixed velocity dispersion than those at $z=0$ (McLeod \& Bechtold 2009; Woo et al. 2008; Greene et al. 2010a), whereas others report no significant evolution with redshift (Shields et al. 2003; Gaskell 2009; Salviander \& Shields 2013). Simi-

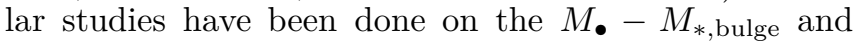


$M_{\bullet}-M_{* \text {,host }}$ relationships, and have argued for evidence supporting an increase in black hole mass at fixed host mass with increasing redshift (Merloni et al. 2010; Bennert et al. 2011). However, it has recently been claimed that these observed trends in the $\mathrm{BH}$ bulge scaling relations may be the result of selection effects in observations of broad line AGN, and that the observations are consistent with a lack of evolution (Schulze \& Wisotzki 2014). Other works have investigated the evolution in the $M_{\bullet}-\sigma$ and $M_{\bullet}$ $M_{* \text {,bulge relations by combining observationally-derived }}$ bulge mass and velocity dispersion functions at different redshifts with predicted SMBH mass functions at the same redshifts. The latter are derived from observed AGN luminosity functions using the Soltan argument and some observationally-motivated assumptions about SMBH merger rates. These studies do not have the same observational biases as those using broad line AGN samples. They find no evolution in the $M_{\bullet}-\sigma$ relation (Shankar et al. 2009a; Zhang et al. 2012), but a larger $M_{\bullet}$ at fixed $M_{* \text {,bulge }}$ with increasing redshift (Zhang et al. 2012).

Because we fix the relations of seed mass to host bulge mass and accreted mass to bulge star formation to be the same at all redshifts, we essentially force the $M_{\bullet}-M_{* \text {,bulge }}$ relation to be constant with redshift. Accounting for ejected and orbiting BHs can cause some evolution in the relation by lowering the central $\mathrm{BH}$ mass relative to the host bulge mass, but, as will be described in \$4.2, the change in mass from these effects is on average fairly small. Also, because we take a fixed relation between galaxy stellar mass and velocity dispersion with a redshift dependence $\sigma \propto(1+z)^{0.47}$, the $M_{\bullet}-\sigma$ relation as a function of redshift is also completely determined by our assumptions, which result in a declining $\mathrm{BH}$ mass at fixed $\sigma$ with increasing redshift. Thus our model cannot reproduce evolution in the $\mathrm{BH}$-galaxy scaling relations. The effect of this on our results is similar to that of assuming an unevolving galaxy bulge mass fraction at fixed stellar mass, as described in \$3.2 however, while assuming a constant bulge mass fraction at all times causes $\mathrm{SMBHs}$ to be too massive at early times, assuming a constant $M_{\bullet}-M_{\text {bulge }}$ relation causes them to be less massive at early times.

Complementary constraints on $\mathrm{BH}$ growth models can be derived from X-ray luminosity functions of AGN. Since X-rays can escape even the most obscured Compton-thick galactic nuclear sources, they offer a unique probe of actively growing $\mathrm{BHs}$ (Salvaterra et al. 2007). Several studies that have attempted to explain the origin of the cosmic X-ray background have also provided insights into both the obscured and unobscured accreting populations of $\mathrm{BHs}$ over cosmic time (c.f. models by Gilli et al. 2007; Treister et al. 2009). Relevant to our study are the constraints obtained by Volonteri et al. (2006) on the accretion history of SMBHs at $z<3$ using observations of the faint X-ray background combined with optical and hard X-ray luminosity functions. They found that a model in which the Eddington ratio is a function of the AGN luminosity - as suggested by previous simulations - fits the observational constraints somewhat better than models with a constant Eddington ratio or an Eddington ratio decreasing with redshift. However, other models have found that the Eddington ratio must depend on both AGN luminosity and redshift in order to produce a high enough AGN fraction at low redshifts to match observations (Merloni \& Heinz 2008; Shankar et al. 2013).

Similarly, Natarajan \& Treister (2009) used the observed AGN X-ray luminosity function from $3<z<0$, combined with a simple model of AGN accretion, to evolve seed black holes. They found that ultra-massive black holes (UMBHs) with masses of $\sim 10^{10} M_{\odot}$ should exist at $z=0$, but that consistency with the observed present-day SMBH mass function requires that there be an upper limit to the masses of SMBHs at about $10^{10} M_{\odot}$. Such an upper limit could be the result of SMBH selfregulation processes, and would result in an evolving slope at the high end of the $M_{\bullet}-\sigma$ relation. They predict an abundance of UMBHs of $\sim 10^{-6}-10^{-7} \mathrm{Mpc}^{-3}$, consistent with extrapolating the $z=0 \mathrm{SMBH}$ mass function to high masses, and propose that UMBHs may be found in central brightest cluster galaxies. Further work on the BHs hosted by central cluster galaxies (CCGs) was done by Volonteri \& Ciotti (2013). SMBHs in such galaxies tend to be overmassive compared to the scaling relations for lower-mass galaxies, although the difference is more pronounced in the $M_{\bullet}-\sigma$ relation than in the $M_{\bullet}-M_{\text {bulge }}$ relation. They created semi-analytic models for the growth of SMBHs in CCGs and found that these trends may be the result of a larger number of dry mergers contributing to the mass of CCGs, because dry mergers increase a galaxy's mass, luminosity, and radius more than its velocity dispersion. Minor mergers can in fact decrease the velocity dispersion of the merged galaxy (Hilz et al. 2012, Figure 9).

As for the population of "wandering" black holes, the buildup of populations of orbiting and ejected black holes as a result of galaxy mergers and gravitational wave recoils has been examined most recently by Rashkov \& Madau (2014). Although their approach bears similarities to ours, they studied the evolution of intermediate-mass black holes (IMBHs), which are thought to be the ancestral seeds of the supermassive black holes currently found in the centers of galaxies. They populated the N-body Via Lactea II cosmological simulation of a Milky Way-size halo (Diemand et al. 2008) with seed IMBHs, which they then allowed to evolve via mergers and gravitational wave recoils. They found that even when assuming "maximal" numbers of BHs would escape the galaxy due to gravitational wave kicks, a sizable population of leftover IMBHs should be orbiting in the halo of a galaxy with the mass of the Milky Way. We focus on slightly different mass scales in this work and examine the central SMBHs and the SMBH wanderers in a typical cluster rather than a galaxy scale halo.

\subsection{Results}

In this section, we present the results of following the SMBH evolution in both the cluster and void environments. We also present some results for the "global average" combination of the cluster and void (\$3.1), intended to be similar to the universe on average.

Figure 1 1 shows the median black hole mass in different categories versus the bulge stellar mass in the void and cluster boxes. As expected given our assumptions for SMBH growth, we reproduce the empirically observed 


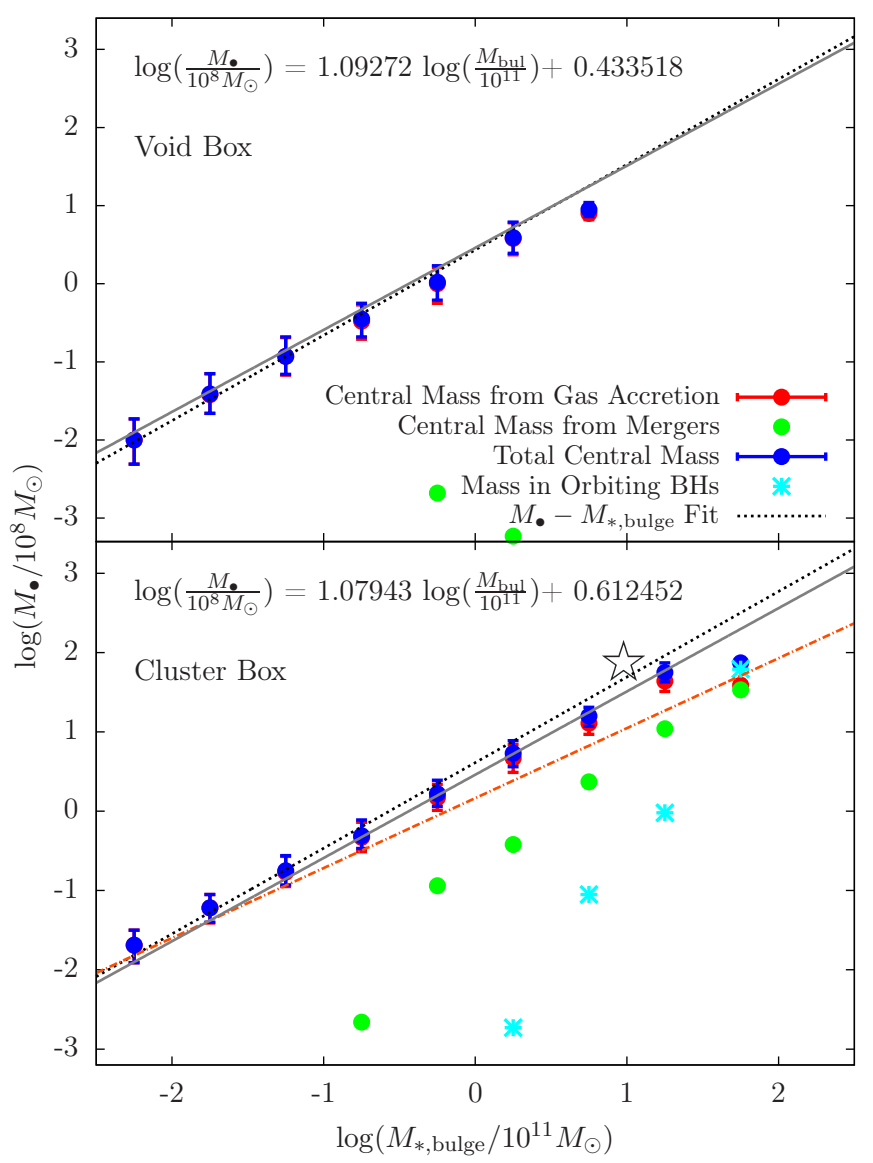

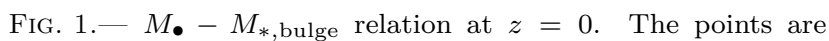
median masses and the error bars represent one quartile. We plot the median $\mathrm{BH}$ mass per galaxy in four categories: the total mass of the central SMBH $\left(M_{\bullet}\right)$, the amount of mass in the central SMBH that was accreted from galactic gas, the amount of central SMBH mass from mergers with less massive SMBHs, and the total amount of mass found in SMBHs that are orbiting in the galaxy. The black dashed line is a fit to $M_{\bullet}-M_{*}$,bulge for all the galaxies in each simulation box (not only the displayed median points), and the resulting relation is displayed on the plot.

The gray solid line is the observationally derived $M_{\bullet}-M_{\text {bulge }}$ relation from McConnell \& Ma (2013). The orange dot-dashed line is a fit to $M_{\bullet}-\sigma$ data from the Virgo cluster from Ferrarese et al. (2006), converted to $M_{\bullet}-M_{*}$ using Equation 5 The star symbol represents the position of M87 and its SMBH (Gebhardt \& Thomas 2009; Forte et al. 2012) marked as a reference.

trend of central black hole mass with bulge stellar mass. Shown for comparison are the $M_{\bullet}-M_{\text {bulge }}$ relation from McConnell \& Ma (2013), and the $M_{\bullet}-M_{*}$ relation from the Virgo cluster (Ferrarese et al. 2006). We find that in the cluster environment for the most massive black holes a larger fraction of their mass growth occurs due to direct black hole mergers compared to lower mass black holes. In the void box, where there are on average fewer mergers, the median contribution of direct mergers to the mass inventory is less than $10^{6} M_{\odot}$ per galaxy at all bulge masses, whereas in the cluster it is $>10^{6} M_{\odot}$ for $M_{*, \text { bulge }}>3 \times 10^{10} M_{\odot}$.

For the same reason, the amount of mass in BHs orbiting in galaxies in the void box is negligible, as opposed to the cluster box, where the orbiting mass is substantial for galaxies with $M_{* \text {,bulge }} \gtrsim 10^{11} M_{\odot}$. In the cluster box, $\sim 2$ orbiting SMBHs with $M_{\bullet} \gtrsim 3 \times 10^{3} M_{\odot}$ are expected for each galaxy with mass $M_{*, \text { bulge }} \approx 10^{11} M_{\odot}$ $\left(M_{*} \approx 1.3 \times 10^{11} M_{\odot}\right)$, with the number increasing for larger masses. For the void box, $\sim 0.3$ orbiting BHs with $M_{\bullet} \gtrsim 3 \times 10^{3} M_{\odot}$ are found in the average galaxy with $M_{* \text {, bulge }} \approx 10^{11} M_{\odot}$. It should be noted that the number of orbiting BHs per galaxy depends highly on the minimum SMBH mass considered, since the dynamical friction time for a SMBH to reach the center of a galaxy is inversely proportional to its mass (Equation 3).

The difference between the fraction of $\mathrm{BH}$ mass contributed by mergers in the void and cluster boxes is shown more clearly in Figure 2 The number-weighted mean mass fraction from mergers is essentially zero for SMBHs with $M_{\bullet}<10^{6} M_{\odot}$ in both boxes and increases for larger masses. In the cluster, the fraction reaches a maximum of $24 \%$ for $M_{\bullet} \approx 10^{10} M_{\odot}$. In the void, the fraction of mass from mergers reaches about $5 \%$ for the most massive BHs. The fraction of central black hole mass from mergers can also be examined as a function of the host bulge mass. The contribution from mergers is negligible for host masses $M_{* \text {,bulge }} \lesssim 10^{9.5} M_{\odot}$ in both the void and cluster boxes. It increases with host mass such that in the void the fraction of $\mathrm{BH}$ mass from mergers is $2 \%$ at $M_{* \text {,bulge }}=10^{10} M_{\odot}, 4 \%$ at $10^{11} M_{\odot}$, and $19 \%$ for the most massive galaxies in the void, which have masses about $10^{12} M_{\odot}$. In the cluster box, the fraction from mergers is $4 \%$ at $M_{* \text {,bulge }}=10^{10} M_{\odot}, 11 \%$ at $10^{11} M_{\odot}$, and $23 \%$ at $10^{12} M_{\odot}$, reaching a maximum of $28 \%$ for the most massive galaxies with $\approx 10^{13} M_{\odot}$. The latter is close to the fraction of galaxy mass attained via mergers by the most massive galaxies in our cluster box, which is $\sim 40 \pm 15 \%$ (Lackner et al. 2012), a value that is significantly lower than that found by some previous simulations (Oser et al. 2010). The fact that the $\mathrm{BH}$ mass fraction from mergers is slightly smaller than that for galaxies is expected due to a combination of two factors. The first is that the mass of our SMBHs is roughly proportional to their host bulge stellar masses, which increase as a fraction of total stellar mass for larger galaxies. Thus the stellar mass ratio of a smaller galaxy to a larger one will tend to be larger than the ratio of the masses of their respective SMBHs, resulting in a larger contribution from mergers for galaxies than for the SMBHs that they host. The other effect is that some fraction of the BHs from merged galaxies are still orbiting at $z=0$. The number-weighted mean mass contribution from mergers for all SMBHs is $0.9 \pm 0.1 \%$ in the void box and $2.4 \pm 0.1 \%$ in the cluster box.

Between redshifts $z=4$ and $z=0$, the mean massweighted merger ratio for SMBH mergers in both the void and cluster is $\sim 1: 5$. Our results can be compared to the median mass-weighted merger ratio of galaxy bulges from Hopkins et al. (2010), who studied galaxy bulges with masses $10^{9} M_{\odot} \lesssim M_{* \text {,bulge }} \lesssim 10^{12} M_{\odot}$ at $z=0$ using semi-empirical models. Our results are consistent with their values for the median merger ratio, which span approximately $1: 6$ to $1: 2$ depending on the final bulge mass. We also compare with the mean mass-weighted merger ratio for individual galaxies found by Oser et al. (2012), who performed cosmological resimulations of galaxies with masses $4.5 \times 10^{10} h^{-1} M_{\odot} \lesssim$ $M_{*} \lesssim 3.6 \times 10^{11} h^{-1} M_{\odot}$ between $z=2$ and $z=0$. Our mean SMBH merger ratios are the same for $2>z>0$ 


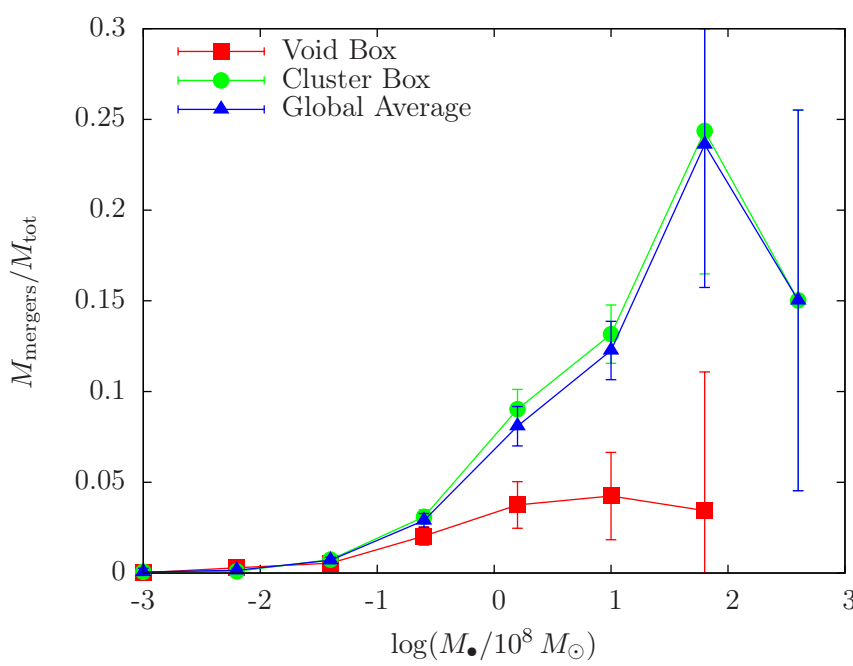

FIG. 2. - The number-weighted mean fraction of total central $\mathrm{SMBH}$ mass gained via $\mathrm{BH}-\mathrm{BH}$ mergers as a function of $\mathrm{SMBH}$ mass at $z=0$. Error bars are $1 \sigma$. Values are shown for BHs in the void and cluster boxes as well as the global average combination of the two.

as for $4>z>0$, and agree with the value of $\sim 1: 5$ obtained by Oser et al. (2012) for galaxies. The agreement with Hopkins et al. (2010) and Oser et al. (2012) is likely a result of the fact that the mean mass-weighted galaxy merger ratio in our simulations is approximately in agreement with those of the referenced works (Lackner et al. 2012). Since SMBH mergers are subsequent to galaxy mergers and SMBHs and their hosts are connected by scaling relations, the similarity of the results is not unexpected.

Figure 3 shows the distribution of central SMBH mass in the form of the fraction of total black hole mass contained in BHs with mass $>M_{\bullet}$. In both the cluster and void box, the total mass is overwhelmingly concentrated in the most massive black holes. At low redshifts, half of the mass is found in SMBHs with $M_{\bullet} \gtrsim 10^{8.5} M_{\odot}$ in the void, and in SMBHs with $M_{\bullet} \gtrsim 10^{9} \widetilde{M}_{\odot}$ in the cluster, despite the fact that such black holes constitute less than $5 \%$ of the total population in both boxes. The effects of $\mathrm{BH}-\mathrm{BH}$ mergers can also be seen, as the fraction of mass contained in high mass black holes increases over time up to $z=0.2$, but then stops as a result of more low mass black holes being added to the population.

A different way of displaying the mass distribution of SMBHs can be seen in Figure 4, which shows the differential mass function per logarithmic SMBH mass interval. Only central SMBHs are included. Unlike Figure 3. in which the fraction of SMBH mass in each bin is normalized to the total SMBH mass in the box, Figure 4 shows the number of SMBHs per unit volume in each mass bin, which also displays the larger number density of SMBHs in the cluster box compared to the void box (which is due to the larger density of galaxies in the cluster). In the top panel, we compare our global average SMBH mass function at $z=0$ to that from observations (Marconi et al. 2004; Shankar et al. 2009b; Shankar 2013), and also show the mass functions of the cluster and void boxes at $z=0$. The lower panel shows the cluster, void, and global average central SMBH mass functions at $z=1$ and $z=1.9$. The number of SMBHs
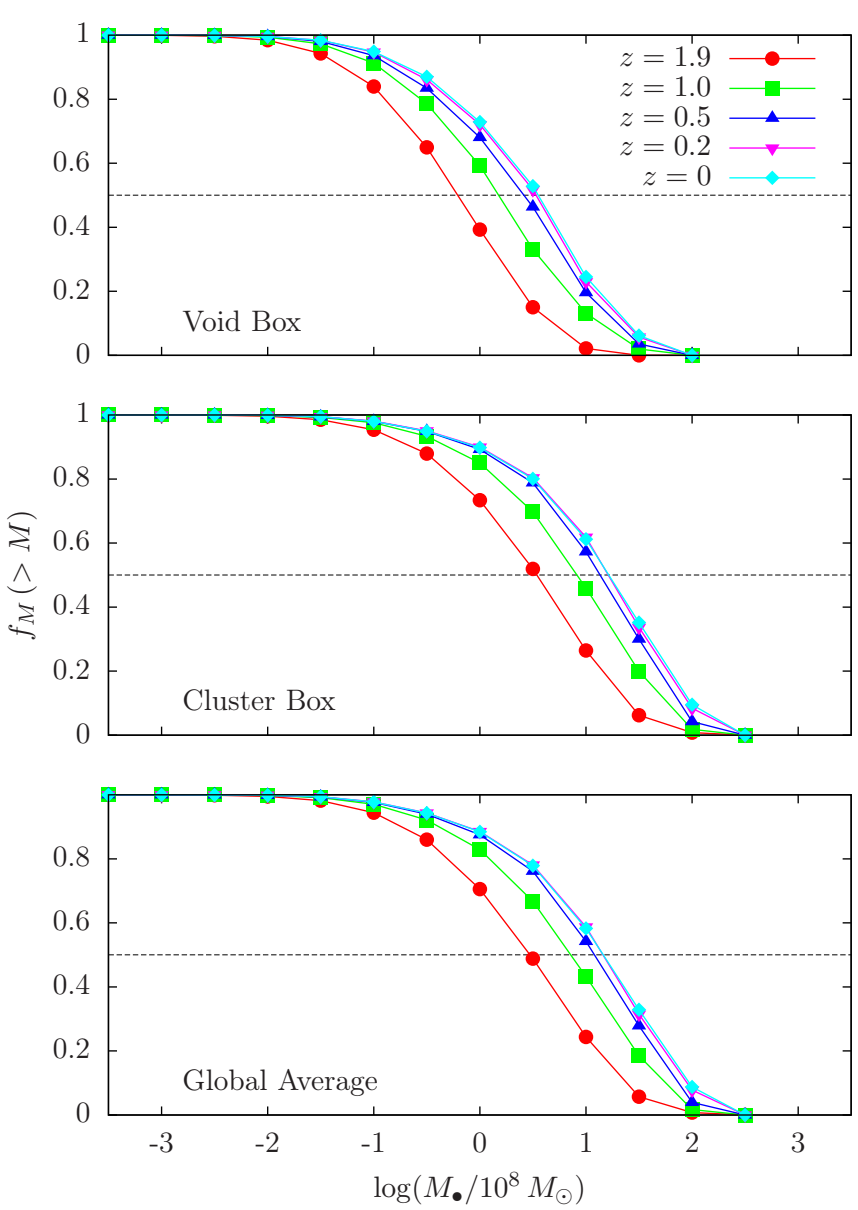

FIG. 3. - The fraction of the total central black hole mass contained in black holes with mass above some $M_{\bullet}$, shown at different redshifts.

increases with time for all $M_{\bullet}>10^{6} M_{\odot}$, but especially for higher masses, in both the cluster and void.

One can see in the top panel of Figure 4 that our $z=0$ mass function is shallower than those of Marconi et al. (2004) and Shankar et al. (2009b), but is nearly in agreement with a more recently derived mass function from Shankar (2013). As described in \$3.1 and \$3.2, we evolve our SMBHs and normalize our SMBH mass densities in the cluster and void under the assumption that the $M_{\bullet}-M_{\text {bulge }}$ relation in McConnell \& Ma (2013) is correct. The mass functions of Marconi et al. (2004) and Shankar et al. (2009b) are derived using a different $M_{\bullet}-M_{\text {bulge }}$ relation, which results in a different shape and lower normalization than our mass function. The mass function from Shankar (2013) is instead derived assuming that all SMBHs follow the $M_{\bullet}-\sigma$ relation of McConnell \& Ma (2013), similar to our assumption. Thus the greater similarity of our mass function to that derived by Shankar (2013) is not surprising.

The growth of SMBHs over time follows different trends in the void and cluster boxes. Figure [5] shows the number-weighted mean rate of mass growth via accretion and mergers per central SMBH as a function of redshift. In both the cluster and void, accretion is the dominant source of growth for SMBHs at all redshifts. Nevertheless, the accretion rate per $\mathrm{BH}$ decreases by a factor of $\sim 5$ and mergers become more significant in both boxes 


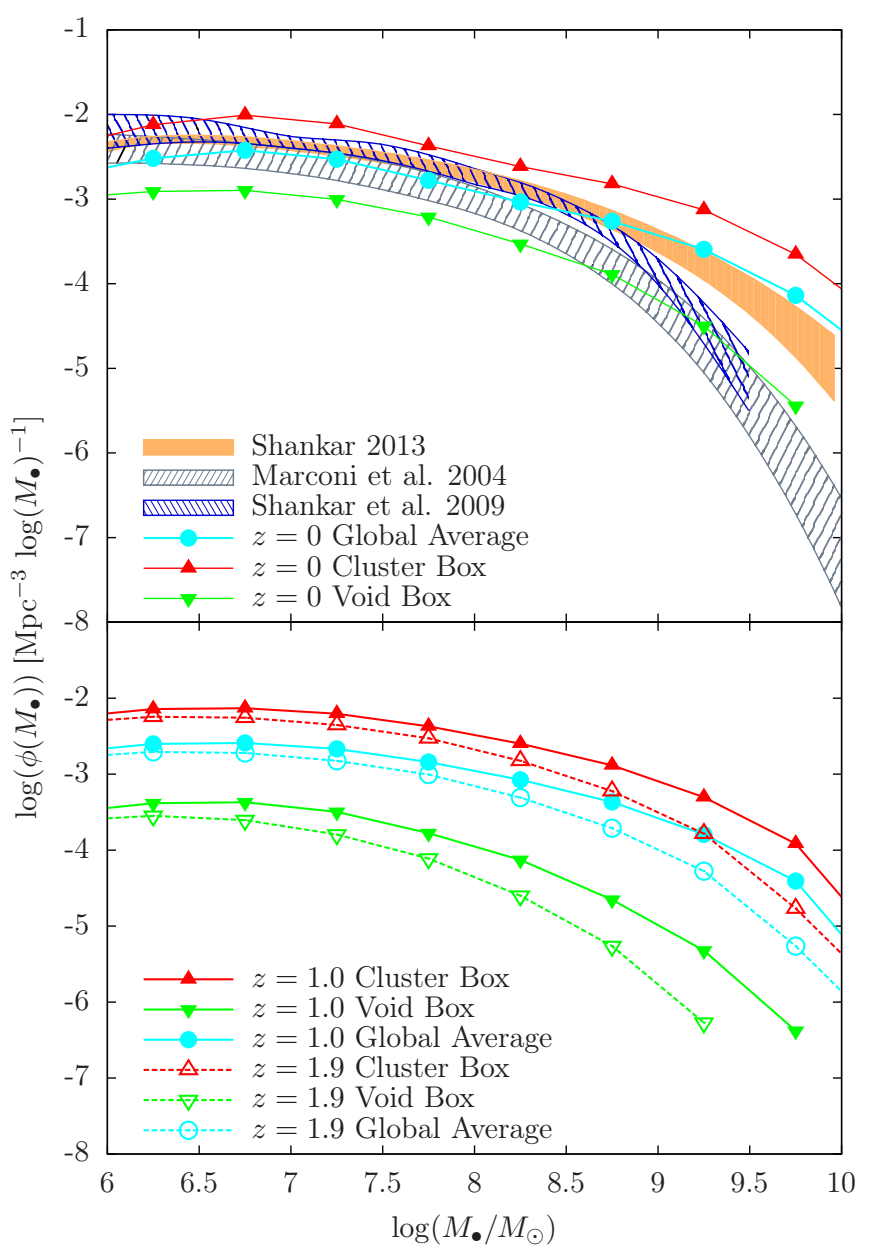

FIG. 4.- Top Panel: The mass function of central SMBHs at $z=0$, for the cluster box, void box, and global average. Also shown for comparison are the observed SMBH mass functions from Marconi et al. (2004), Shankar et al. (2009b), and Shankar (2013). Bottom Panel: Same as the top panel, but at $z=1$ and $z=1.9$.

between $z=4$ and $z=0$. While the mean accretion rate per SMBH is only slightly higher in the cluster than in the void, the mass growth rate from mergers is $\gtrsim 10$ times higher in the cluster at all redshifts.

Because mergers become more significant over time, in both the void and cluster the total black hole mass from mergers is added slightly later on average than that from accretion. In the void, half of the total mass from accretion at $z=0$ has been added by $z \approx 0.6$, whereas for mergers half the mass has been added by $z \approx 0.5$. In the cluster, both half the merged and accreted mass are added earlier than in the void, and the difference between the two is larger: half the accreted mass is added by $z \approx 1.0$, while half the merged mass is added after $z \approx 0.7$. These SMBH growth trends parallel those of galaxies. Galactic mass assembly in the cluster tends to happen earlier than in the void, and in both the cluster and void mass buildup by accretion peaks at earlier times than by mergers (Lackner et al. 2012).

The connection between galaxy growth via galaxy mergers and $\mathrm{BH}$ growth via $\mathrm{BH}-\mathrm{BH}$ mergers can also be seen in the top panel of Figure 6. This panel shows the number of mergers, weighted by the merger mass ratio (of BHs or bulges), per central BH per Gyr. The merger

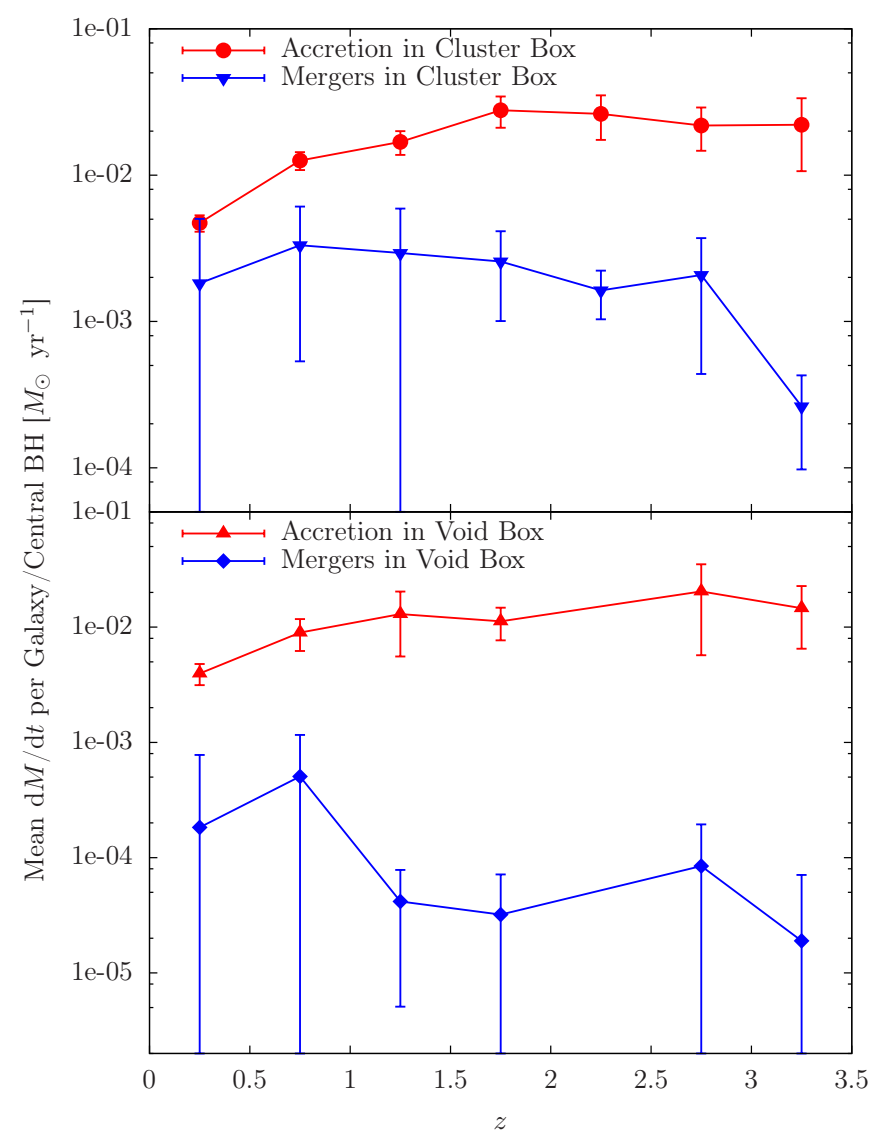

FIG. 5.- Number-weighted mean mass growth rate per central SMBH from accretion and mergers. The top and bottom panels show the cluster and void box, respectively. The red lines show the rate of mass increase due to gas accretion onto central black holes; the blue lines show the rate of mass increase due to mergers with smaller black holes. The mean mass of a SMBH at $z=3.25$ is $4.5 \times 10^{6} M_{\odot}$ in the cluster and $1.4 \times 10^{6} M_{\odot}$ in the void, increasing to $9.3 \times 10^{7} M_{\odot}$ in the cluster and $2.5 \times 10^{7} M_{\odot}$ in the void at $z=0.25$.

history of the $\mathrm{BH}$ population approximately follows that of the bulge population — which is to be expected, as $\mathrm{BH}$ mergers are subsequent to galaxy mergers, although sometimes delayed by dynamical friction. One can also see in this panel that in the cluster on average a central $\mathrm{BH}$ is predicted to experience a mass increase due to mergers equivalent to a $\sim 1: 10$ merger per Gyr at $z \sim 3$, declining to the equivalent of $\mathrm{a} \sim 1: 100$ merger per Gyr at $z \sim 0$. In the void, the mass gain rate from mergers is lower than that in the cluster but declines with time more slowly, becoming the same as for the cluster at $z \sim 0$. We predict a mass gain in the void per average central $\mathrm{BH}$ equivalent to a $\sim 1: 50$ merger per Gyr at $z \sim 3$ and $\mathrm{a} \sim 1: 100$ merger per Gyr at $z \sim 0$. There is a factor of $\sim 3$ difference between the void and cluster in the fraction of growth due to mergers per central galaxy for $z \gtrsim 0.5$; this is smaller than the factor of $\gtrsim 10$ seen in Figure 5 for $d M / d t$ from mergers because SMBHs in the void are on average less massive than those in the cluster.

The bottom panel of Figure 6] shows a related quantity, the fraction of central BHs ejected from their host galaxies per Gyr as a function of redshift. This follows the trend of the $\mathrm{BH}$ merger rate closely, since mergers 


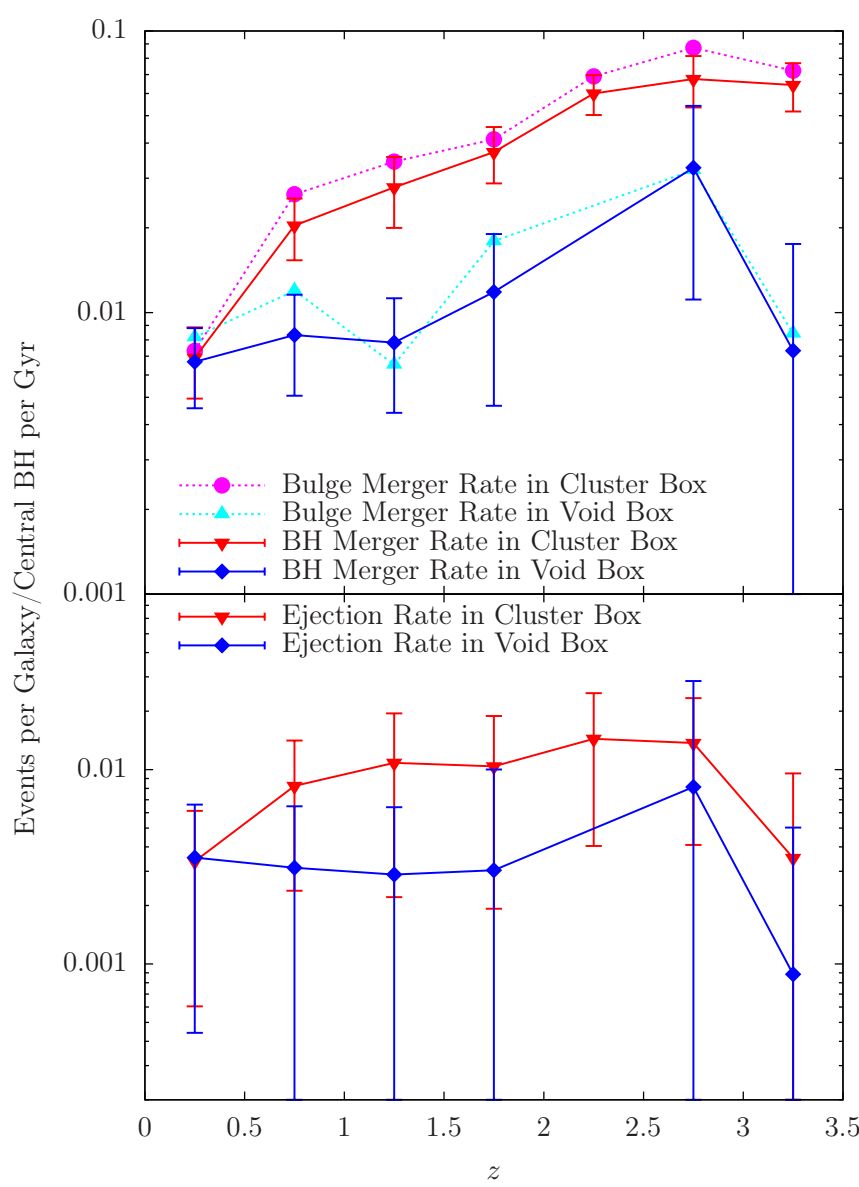

FIG. 6. - Top Panel: Merger rates of galaxy bulges and SMBHs, expressed as the number of mergers per galaxy or central $\mathrm{BH}$, weighted by merger ratio (e.g., a merger with ratio $1: 2$ is counted as $1 / 2$ ), per Gyr. Dotted lines represent the merger rate for bulges and solid lines represent the merger rate for black holes.

Bottom Panel: The ejection rate of black holes from their host galaxies, expressed as the number of ejections per Gyr.

cause ejections via gravitational wave recoil. The scatter is very large in both the cluster and void, but the fraction of BHs ejected per Gyr is fairly constant over time: in the cluster it is $\sim 0.01$, and in the void, $\sim 0.003$.

The velocity of the $\mathrm{BH}$ recoil is sensitively dependent on the angles that the spins of the merging $\mathrm{BHs}$ make with the angular momentum of their orbit. When these spins are aligned, the kick velocity cannot exceed $200 \mathrm{~km}$ $\mathrm{s}^{-1}$ (Bogdanović et al. 2007). However, even though we assume an isotropic distribution of spin vectors, the fraction of ejected BHs is relatively small, as can be inferred from Figure 6 and will be described in further detail in $\$ 4.2 .2$. As a result, even a complete lack of ejections will not have a large effect on the remaining results. If we assume that all merging BHs are aligned to within $\sim 10^{\circ}$ of the orbital angular momentum, as expected for gas-rich mergers in cold disks (Dotti et al. 2010), generally no ejections occur in our realizations. Despite the smaller kicks out of the center of the galaxy, the number and mass in orbiting BHs is not significantly affected. Relaxing the assumption to take all BHs to be aligned within a larger angle of $\sim 30^{\circ}$, as in gas-rich mergers in warm disks (Dotti et al. 2010), we find that the fraction of BHs and $\mathrm{BH}$ mass ejected is roughly half that when assuming

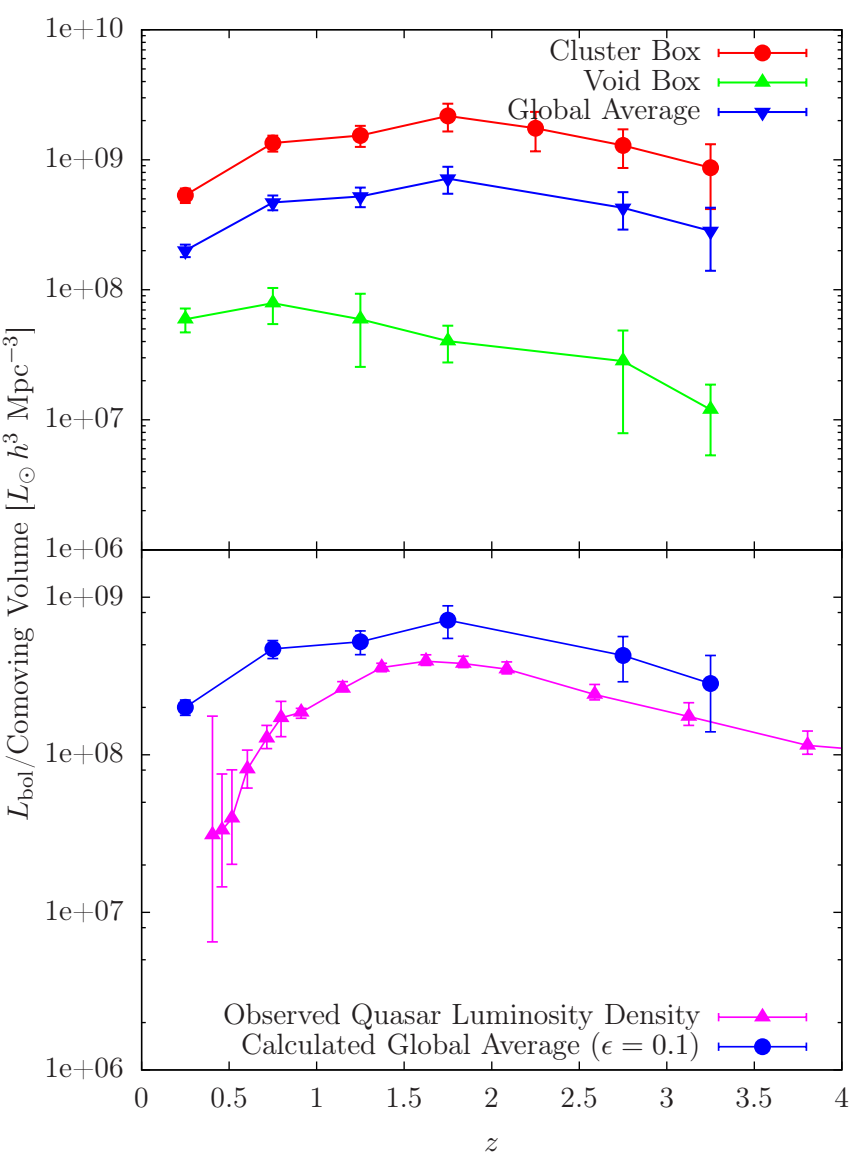

FIG. 7.- Top Panel: Estimated SMBH bolometric luminosity $E=0.1 \dot{M}_{a c c} c^{2}$ per unit comoving volume due to gas accretion as a function of $z$ for the void and cluster boxes and their weighted global average.

Bottom Panel: In magenta is the observed QSO luminosity density from Hopkins et al. (2007). The blue solid line is our calculation for the global average bolometric luminosity using $\epsilon=0.1$, also shown in the top panel.

an isotropic distribution of spins. The population of orbiting BHs is unaffected. Thus, our assumptions about the directions of $\mathrm{BH}$ spins do not appear to affect our results significantly.

Figure 7 shows the expected bolometric luminosity density of SMBHs due to gas accretion as a function of redshift for the cluster and void boxes as well as the global average. We assume a mass to energy conversion efficiency of $\epsilon=0.1$, so that $L_{\mathrm{bol}}=0.1 \dot{M}_{\mathrm{acc}} c^{2}$. The results can be scaled for other assumed values of the efficiency. In the lower panel of Figure 7, we compare our estimated global average luminosity density to the observed QSO luminosity density from Hopkins et al. (2007). The luminosity density we calculate is higher than that of Hopkins et al. (2007). The mass to energy conversion efficiency we use is consistent with that found in $\mathrm{Yu}$ \& Tremaine (2002), but their calculation assumed a local black hole mass density of $\rho_{\bullet}=2.5 \times 10^{5} M_{\odot}$ $\mathrm{Mpc}^{-3}$, whereas we use $9.1 \times 10^{5} M_{\odot} \mathrm{Mpc}^{-3}$, which would imply a lower value of $\epsilon$. Thus the fact that our calculated luminosity density is higher than that observed is not surprising.

Because our adopted $\rho_{\bullet}$ implies a lower radiative efficiency from the Soltan argument, it is more interesting 
to look at the difference in shape of our SMBH luminosity density with redshift as opposed to normalization. The global average luminosity output we find is roughly constant with redshift for $z>0.5$, decreasing for the most recent times. However, the downturn we find at low redshift is much smaller than that seen in observations. This may be because the cosmological simulation we use is known to especially overproduce stars at low $z$ (Lackner et al. 2012), which would cause the BH accretion rate to remain too high as well. Our scaling of the void to cluster is also quite simplistic, taking the void to cluster ratio to be constant with redshift. If this is not the case, the shape of our predicted trend would also change.

Figure 8 shows our global average instantaneous bolometric SMBH luminosity function (LF) at redshifts 2.0, $1.0,0.5$, and 0.1 . For comparison are shown model fits to observational data from Hopkins et al. (2007). In Figure [7] it can be seen that the total luminosity output we predict from QSOs is higher than that found by Hopkins et al. (2007) and Shankar et al. (2009b), especially at $z<0.5$. Figure 8 shows the instantaneous LF as opposed to the mean total luminosity in broader redshift bins shown in Figure 7, but the integrated total luminosity we predict is still larger than that obtained from the LFs of Hopkins et al. (2007) and Shankar et al. (2009b), especially at $z=0.1$.

Also, our LF is not of the same shape as that observed; it is skewed towards low luminosities. This is because our simple assumptions for the $\mathrm{SMBH}$ growth, combined with the limitations of our simulation, are unable to produce very high luminosity QSOs and assign luminosities that are too low to some fraction of SMBHs instead. We assume that the instantaneous $\mathrm{SMBH}$ accretion rate is proportional to the instantaneous star formation rate of its host, and that there is a constant proportionality between the instantaneous SMBH accretion rate and the instantaneous luminosity, such that the latter is always $\epsilon=0.1$ times the former. Our model includes no detailed assumptions about accretion physics onto the $\mathrm{SMBH}$ that could cause large variability in the SMBH luminosity. Furthermore, our simulation models the star formation rate as exponentially declining for each star particle (Cen \& Ostriker 1992), so it does not capture the observed "bursty" nature of star formation, which would increase the number of high-luminosity QSOs during bursts in the simple scheme described above. Thus our model has no mechanism for producing the brightest QSOs. If we included the mechanisms above, some of the SMBHs in our model would be assigned higher luminosities, which would increase the number of highluminosity QSOs relative to low-luminosity ones and decrease the discrepancy in LF shape between our results and observations.

We also calculate the expected total energy density emitted in gravitational wave radiation as a result of $\mathrm{BH}-\mathrm{BH}$ mergers. The results are shown in Figure 9 for the global average. The luminosity follows a general increase with time, with the increase being significantly steeper for $z \gtrsim 2.5$. The gravitational wave luminosity, while very similar as a function of redshift to the rate of mass increase due to mergers, is not directly proportional, since the energy in emitted gravitational wave radiation is also dependent on the mass ratio of the merging

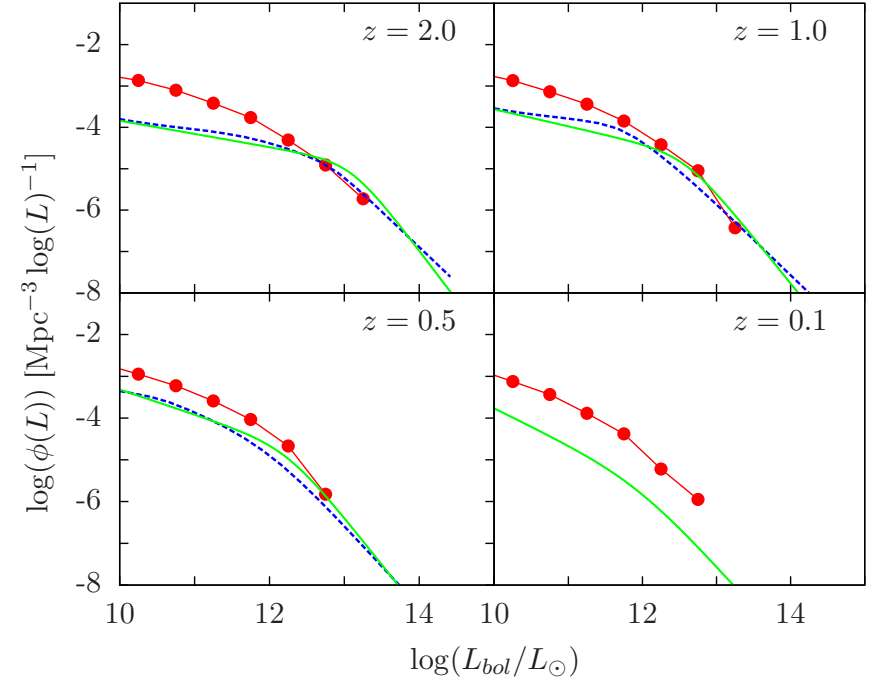

FIG. 8.- Global average instantaneous bolometric SMBH luminosity functions at $z=2.0,1.0,0.5$ and 0.1 (red points) compared with SMBH bolometric luminosity functions derived from observations from Hopkins et al. (2007) (green lines) and Shankar et al. (2009b) (blue dashed lines; for $z \geq 0.5$ only).

black holes (see Equations 6] 10).

Further, we calculate the gravitational wave strain produced by our population of black holes using the method described in Sesana (2013b). For a population of merging black hole binaries, where the black holes in each binary have masses $M_{1}$ and $M_{2}$ with $M_{1}>M_{2}$ and $q \equiv M_{2} / M_{1}$, the characteristic amplitude of the gravitational wave signal $h_{c}$ is given by

$h_{c}^{2}(f)=\frac{4}{\pi f} \iiint \mathrm{d} z \mathrm{~d} M_{1} \mathrm{~d} q \frac{\mathrm{d}^{3} n}{\mathrm{~d} z \mathrm{~d} M_{1} \mathrm{~d} q} \frac{1}{1+z} \frac{\mathrm{d} E_{\mathrm{gw}}(\mathcal{M})}{\mathrm{d} \ln f_{r}}$

where the energy emitted per logarithmic frequency interval is

$$
\frac{\mathrm{d} E_{\mathrm{gw}}}{\mathrm{d} \ln f_{r}}=\frac{\pi^{2 / 3}}{3} \mathcal{M}^{5 / 3} f_{r}^{2 / 3}
$$

where $\mathcal{M}=\left(M_{1} M_{2}\right)^{3 / 5} /\left(M_{1}+M_{2}\right)^{1 / 5}$ is the chirp mass of the binary and $f_{r}=(1+z) f$ is the rest frame frequency of the gravitational radiation. The amplitude $A$ is defined by

$$
h_{c}(f)=A\left(\frac{f}{\mathrm{yr}^{-1}}\right)^{-2 / 3} .
$$

We find a global average strain amplitude of $\log A=$ $-14.7 \pm 0.1$ ( $1 \sigma$ error $)$, or $A=2.0 \times 10^{-15}$. This is below the current observational upper limit of $A=$ $6 \times 10^{-15}$ found by van Haasteren et al. (2011). Our result is consistent with the $1 \sigma$ range found by Sesana $(2013 \mathrm{a}, \mathrm{b})$ for models using the $M_{\bullet}-M_{\text {bulge }}$ relation of McConnell \& Ma (2013) as we do. Our results are within the $2 \sigma$ lower limit of McWilliams et al. (2014) but lower than their main result of $A=4.1 \times 10^{-15}$. This is probably because McWilliams et al. (2014) computed the merger rate assuming all late-time growth to be due to mergers, whereas in our model late-time growth is still dominated by accretion (Figure 5). For a $f^{-2 / 3}$ gravitational wave spectrum from inspiraling binaries, this value for the strain could potentially be detected with 


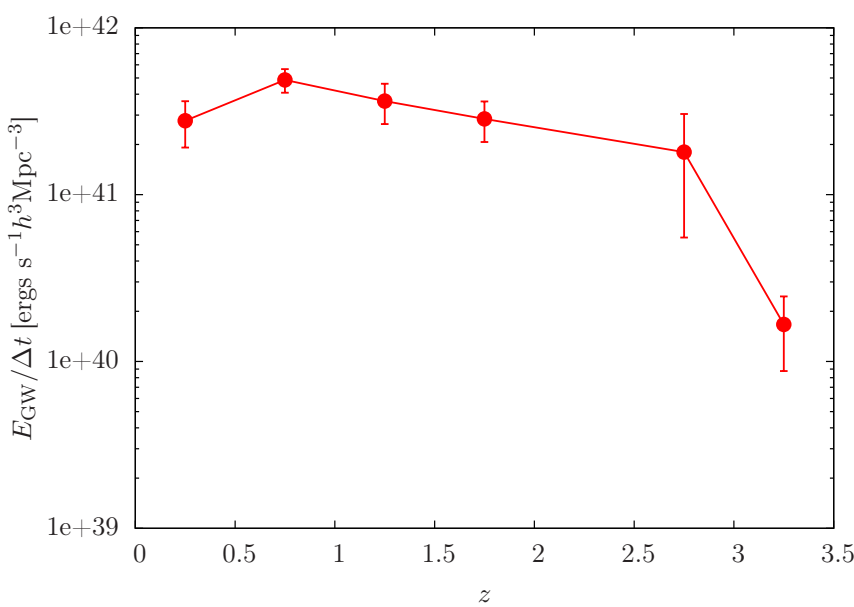

FIG. 9.- The global average gravitational wave luminosity density resulting from $\mathrm{BH}-\mathrm{BH}$ mergers.

$\lesssim 8$ years of pulsar timing observations at current sensitivities, or fewer with higher sensitivities (Sesana 2013a, Figure 2).

It should be noted that our simulations suffer from overmerging of galaxies (Lackner et al. 2012). Also, because the galaxies in our simulation have higher stellar masses than the scaled-down ones we use for our calculation, our galaxy merger rates are further overestimated. This is apparent from the dependence on stellar mass of the dynamical friction time for a galaxy in a subhalo to merge with the central galaxy in an NFW dark matter halo; for this case $t_{\mathrm{DF}} \propto\left(1+M_{*} / M_{\mathrm{DM}}\right)^{-9}$ (McWilliams et al. 2012). This may lead to an overestimation of the number of $\mathrm{BH}-\mathrm{BH}$ mergers, which would cause us to overestimate the gravitational wave luminosity and strain. It would also increase the predicted number of orbiting and ejected SMBHs; these results are described further below.

\subsubsection{Scatter in the $M_{\bullet}-M_{* \text {,bulge }}$ relation with mass}

We divide the galaxies in the cluster and void boxes at $z=0$ into bins in bulge stellar mass, and fit separate

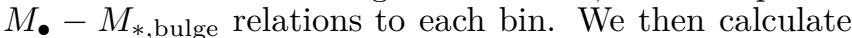
the scatter around the relation in each bin, $\sigma$, given by

$$
\sigma^{2}=\frac{\sum_{i}\left[\log _{10}\left(M_{\bullet}, i\right)-\alpha-\beta x_{i}\right]^{2}}{N_{\text {dof }}},
$$

where $\alpha$ and $\beta$ are the coefficients of the fit. We plot the scatter for each bin in Figure 10. The relation is found to be tighter for black holes with larger masses in both the void and cluster box. The current sample of SMBHs with observationally measured masses is not sufficiently large to determine whether a such a decrease is seen in the scatter. The most complete sample to date is found in McConnell \& Ma (2013), who calculate the intrinsic scatter in the $M_{\bullet}-M_{\text {bulge }}$ relation using several different methods. In Figure [10] we plot their estimates for the intrinsic scatter from Bayesian fits to separate mass bins, as this method is the most similar to ours because it involves fitting the individual mass bins with separate $M_{\bullet}-M_{\text {bulge }}$ relations. The values of the scatter we obtain are slightly in tension with those found by McConnell \& Ma (2013), being significantly smaller than

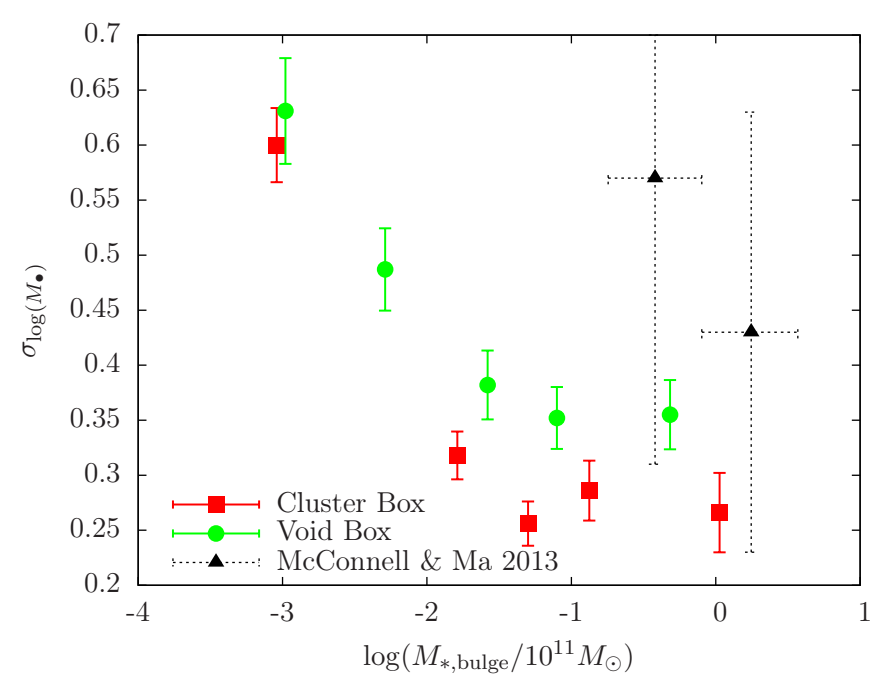

FIG. 10. - Scatter around the $M_{\bullet}-M_{*}$ bulge relation as a function of $M_{*, \text { bulge }}$ for the cluster and void boxes at $z=0$. Points represent the scatter $\sigma$ around a separate $M_{\bullet}-M_{*}$,bulge relation fit in each bin. Error bars are one standard deviation of $\sigma$. Also plotted as black triangles with dotted error bars are the values of the intrinsic scatter from Bayesian fits to the $M_{\bullet}-M_{\text {bulge }}$ relation in separate mass bins from McConnell \& Ma (2013).

their mean values for the intrinsic scatter but marginally consistent with their large error bars.

A decrease in scatter with increasing bulge or $\mathrm{BH}$ mass has been proposed as an expected result of galaxy and subsequent $\mathrm{BH}-\mathrm{BH}$ mergers, which tighten the black hole scaling relations due to the central limit theorem (Hirschmann et al. 2010). However, in our model, mergers contribute only a small amount to this decrease in scatter. The majority of the decrease originates from the fact that more massive SMBHs tend to have had more episodes of accretion, which, because it is proportional to the star formation in the host galaxy bulge, also lowers the scatter in the $M_{\bullet}-M_{*, \text { bulge }}$ relation by the central limit theorem. Because our merger trees have only a few discrete time slices at which we grow every existing $\mathrm{SMBH}$ via accretion, the most massive SMBHs are also those that were seeded the earliest. However, this is simply a limitation of our model, and the fact that BHs which experience more star formation-related accretion episodes will be both more massive and have tighter BHgalaxy scaling relations is true independent of the length of time a SMBH has existed.

\subsubsection{Corrections to the Soltan argument}

As described in \$2, the Soltan argument depends on our ability to accurately measure the mass density in SMBHs at the present day. Any luminosity emitted via the accretion of mass onto an SMBH will be included in the inventory of total SMBH luminosity produced over all time. However, if some fraction of SMBHs end up orbiting or ejected by the present day, some fraction of the mass accreted onto SMBHs will not be included in an inventory of local SMBH mass density, which only counts the SMBH mass in galactic centers. The fraction of SMBH luminosity produced by the accretion of such mass will be assumed to have come from the SMBHs that are found in the centers of galaxies, causing their mean radiative efficiency $\epsilon$ to be overestimated. In our 
model, we keep track of the fraction of mass in orbiting and ejected BHs that must be a correction to the Soltan argument. We present these fractions below. We also give ranges for some of the results we obtain in multiple realizations of the model to emphasize that some of these results can vary widely between our different realizations and so should be taken as uncertain.

We find that at $z=0$, in the void, the fraction of all $\mathrm{BHs}$ that are orbiting in the outskirts of their host galaxies is $3.2 \pm 0.7 \%$, with a range between our different realizations of $1.6 \%$ to $5.0 \%$. This includes all BHs with masses $M_{\bullet} \gtrsim 3 \times 10^{3} M_{\odot}$. The fraction of the total SMBH mass in orbiting black holes is $0.11 \pm 0.06 \%$, with a large range $0.007 \%$ to $0.24 \%$. For the cluster, the amount of orbiting mass is much greater than in the void, as can already be seen in Figure 11. In fact, there is a very large population of orbiting SMBHs in the cluster, with $40.4 \pm 0.4 \%$ of BHs at $z=0$ orbiting; however, most of these BHs are quite small (since less massive $\mathrm{BHs}$ have longer dynamical friction times), and so the fraction of mass in orbit is $7.6 \pm 1.8 \%$, with range $4.3 \%$ to $13.9 \%$. It should be noted that these orbiting black holes are highly concentrated in the most massive galaxies in our cluster box - as could be expected from the fact that a larger galaxy will have a longer dynamical friction time. In fact, approximately one third of all orbiting BHs in the cluster are found in the most massive galaxy. Combining the cluster and void, these values correspond to a global average fraction of orbiting $\mathrm{BHs}$ of $35.7 \pm 0.4 \%$. The fraction of mass that is unaccounted for by observing galaxy centers is $7.0 \pm 1.6 \%$, with range $4.0 \%$ to $12.7 \%$.

We also predict the fraction of black holes that are completely ejected from their host galaxies due to gravitational wave recoil. At $z=0$, we find that in the void $2.3 \pm 0.7 \%$ of all black holes are not associated with any galaxy as a result of being ejected, equivalent to a loss of $2.2 \pm 2.2 \%$ of the total SMBH mass with very large range $0.1 \%$ to $9.9 \%$. In the cluster $4.1 \pm 0.5 \%$ of all $\mathrm{BHs}$ and $4.4 \pm 3.2 \%$ (range $1.2 \%$ to $13.9 \%$ ) of all $\mathrm{BH}$ mass is ejected. It should be noted that while the fraction of black holes ejected is nearly constant for all our runs of the model, the fraction of mass ejected can vary significantly from one to the other. The global average fraction of ejected $\mathrm{BHs}$ is $3.9 \pm 0.4 \%$, and the fraction of mass ejected is $4.2 \pm 2.9 \%$ with a range $1.2 \%$ to $12.8 \%$.

In combination, we predict that the total correction to the Soltan argument from both unaccounted-for orbiting and ejected $\mathrm{BHs}$ is within the range $5.9-20.5 \%$, on average $11.2 \pm 3.4 \%$.

\section{CONCLUSIONS}

We have used the results of a set of hydrodynamical cosmological simulations of a void and cluster region in the universe to predict the evolution of the supermassive black holes that reside in the galaxies in these regions. We find significant late time growth of black holes in massive galaxies, although this growth may be exaggerated in our simulation. Our predicted $M_{\bullet}-M_{* \text {, bulge }}$ relation agrees well with observed trends, as might be expected since our accretion rate onto the black holes was set to reproduce the $z=0 \mathrm{SMBH}$ mass densities in cluster and void regions determined from observations. We calculate the contribution to the mass of each central black hole from accretion of gas and mergers with smaller black holes subsequent to the mergers of their host galaxies. We find that in the cluster, the total $\mathrm{BH}$ mass from mergers is added later on average than the mass from accretion, with half the mass from accretion added before $z \approx 1.0$ and half the mass from mergers added after $z \approx 0.7$. In the void, half the total mass accreted onto the $\mathrm{BH}$ population is accreted before $z \approx 0.6$, but half the merged mass is added after $z \approx 0.5$. Mergers contribute a negligible amount to the mass of black holes with $M_{\bullet} \lesssim 10^{6} M_{\odot}$ in both the cluster and the void region. In the void, the number-weighted mean fraction of mass from mergers rises with black hole mass up to $\approx 5 \%$ for BHs with $M_{\bullet} \gtrsim 10^{8} M_{\odot}$. In the cluster, the fraction from mergers reaches a maximum value of $23 \%$ for $M_{\bullet} \approx 10^{10} M_{\odot}$. The mean fraction of mass from mergers is larger in the cluster box than for galaxies of the same mass in the void box, although values for highmass BHs have large scatter (Figure 2).

Additionally, we predict the mass in black holes orbiting in galaxies due to a galaxy-galaxy merger or gravitational wave recoil. While essentially negligible in the void, a significant amount of such mass is expected in cluster galaxies with $M_{* \text {,bulge }} \gtrsim 10^{11} M_{\odot}$ at $z=0$. In the cluster, $40 \%$ of the $\mathrm{BHs}$ by number and $7.6 \%$ of the $\mathrm{BH}$ mass is orbiting. We predict around 2 orbiting black holes on average for a galaxy in the cluster with bulge mass around $10^{11} M_{\odot}$, or total stellar mass around $1.3 \times 10^{11} M_{\odot}$. Such orbiting black holes are expected to produce observational signatures such as stellar tidal disruption flares that are off-center in the galaxy (Komossa \& Merritt 2008; Li et al. 2012; Liu \& Chen 2013). They are also a candidate to explain via gaseous accretion observed ultra-luminous Xray sources (Islam et al. 2004; Volonteri \& Perna 2005; McWilliams et al. 2014).

We compute the expected energy emitted in gravitational wave radiation due to black hole mergers, shown in Figure 9. More energy is expected at smaller redshifts due to the larger amount of mass added via SMBH mergers during this time. We calculate the total strain amplitude from gravitational waves to be $\log A=-14.7 \pm 0.1$. This is potentially observable by pulsar timing arrays (McWilliams et al. 2014; Sesana 2013a). We also compute the bolometric luminosity from accretion, shown in Figure 7. which is directly proportional to the mass increase from accretion.

We keep track of both SMBHs that end up orbiting in a galaxy due to insufficient dynamical friction and SMBHs that are ejected from their hosts by gravitational wave recoils. These two populations are not accounted for in attempts to calculate the local mass density of SMBHs by measuring the masses of black holes at the centers of galaxies. As such, they are a correction to the Soltan argument. We find that such SMBHs constitute between $6 \%$ and $21 \%$ of the total mass in SMBHs, with a mean of $11.2 \pm 3.4 \%$. Thus the efficiency of accretion in producing observable energy output calculated by the Soltan argument should be reduced by the factor $0.888 \pm$ 0.034 .

We also find a decreasing variance around the $M_{\bullet}-$ $M_{*, \text { bulge }}$ relation with increasing mass in both the cluster and the void, shown in Figure [10. This is a result of the fact that more massive SMBHs have undergone more accretion episodes, which tighten the scaling relations due 
to the central limit theorem. Although current observational data are not sufficient to confirm or disprove the existence of a decrease in scatter, our results are consistent with the latest observational findings within the errors.

Therefore, late time mergers and their environment have interesting and observationally detectable consequences for the mass assembly history of supermassive black holes. The prediction that we make of a significant population of orbiting SMBHs in massive cluster galaxies is testable by future observations.
Computing resources were in part provided by the NASA High-End Computing (HEC) Program through the NASA Advanced Supercomputing (NAS) Division at Ames Research Center. This work is supported in part by grant NASA NNX11AI23G. This work was also supported by World Premier International Research Center Initiative (WPI Initiative), MEXT, Japan. A.K. is supported by the National Science Foundation Graduate Research Fellowship, Grant No. DGE-1148900. P.N. acknowledges support from a NASA-NSF TCAN award number 1332858 .
Avila-Reese, V., Zavala, J., \& Lacerna, I. 2014, MNRAS, 441, 417 Baker, J. G., Boggs, W. D., Centrella, J., et al. 2008, ApJ, 682, L29

Barausse, E. 2012, MNRAS, 423, 2533

Barausse, E., Morozova, V., \& Rezzolla, L. 2012, ApJ, 758, 63

Behroozi, P. S., Conroy, C., \& Wechsler, R. H. 2010, ApJ, 717, 379

Beifiori, A., Courteau, S., Corsini, E. M., \& Zhu, Y. 2012, MNRAS, 419, 2497

Bekenstein, J. D. 1973, ApJ, 183, 657

Belli, S., Newman, A. B., \& Ellis, R. S. 2014, ApJ, 783, 117

Bennert, V. N., Auger, M. W., Treu, T., Woo, J.-H., \& Malkan, M. A. 2011, ApJ, 742, 107

Bernardi, M., Meert, A., Sheth, R. K., et al. 2013, MNRAS, 436, 697

Bernardi, M., Roche, N., Shankar, F., \& Sheth, R. K. 2011a, MNRAS, 412, 684

- 2011b, MNRAS, 412, L6

Bezanson, R., van Dokkum, P. G., van de Sande, J., et al. 2013, ApJ, 779, L21

Binney, J., \& Tremaine, S. 1987, Galactic dynamics

Bogdanović, T., Reynolds, C. S., \& Miller, M. C. 2007, ApJ, 661, L147

Bolzonella, M., Kovač, K., Pozzetti, L., et al. 2010, A\&A, 524, A76

Booth, C. M., \& Schaye, J. 2010, MNRAS, 405, L1

-. 2011, MNRAS, 413, 1158

Bournaud, F., Dekel, A., Teyssier, R., et al. 2011, ApJ, 741, L33

Bournaud, F., Juneau, S., Le Floc'h, E., et al. 2012, ApJ, 757, 81

Brinchmann, J., Charlot, S., White, S. D. M., et al. 2004, MNRAS, 351, 1151

Brusa, M., Fiore, F., Santini, P., et al. 2009, A\&A, 507, 1277

Bryan, G. L. 1999, Comput. Sci. Eng., Vol. 1, No. 2, p. 46 - 53, 1 , 46

Campanelli, M., Lousto, C., Zlochower, Y., \& Merritt, D. 2007a, ApJ, 659, L5

Campanelli, M., Lousto, C. O., Zlochower, Y., \& Merritt, D. 2007b, Physical Review Letters, 98, 231102

Cen, R. 2011a, ApJ, 741, 99

-. 2011b, ApJ, 742, L33

-. 2012a, ApJ, 753, 17

—. 2012b, ApJ, 748, 121

-. 2013, ApJ, 770, 139

Cen, R., Kang, H., Ostriker, J. P., \& Ryu, D. 1995, ApJ, 451, 436

Cen, R., Nagamine, K., \& Ostriker, J. P. 2005, ApJ, 635, 86

Cen, R., \& Ostriker, J. P. 1992, ApJ, 399, L113

Chen, C.-T. J., Hickox, R. C., Alberts, S., et al. 2013, ApJ, 773, 3

Choi, E., Naab, T., Ostriker, J. P., Johansson, P. H., \& Moster,

B. P. 2014, MNRAS, 442, 440

Cibinel, A., Carollo, C. M., Lilly, S. J., et al. 2013, ApJ, 776, 72

Ciotti, L., \& Ostriker, J. P. 1997, ApJ, 487, L105

Cirasuolo, M., Shankar, F., Granato, G. L., De Zotti, G., \& Danese, L. 2005, ApJ, 629, 816

Cisternas, M., Jahnke, K., Bongiorno, A., et al. 2011, ApJ, 741, L11

Di Matteo, T., Colberg, J., Springel, V., Hernquist, L., \& Sijacki, D. 2008, ApJ, 676, 33

Di Matteo, T., Croft, R. A. C., Springel, V., \& Hernquist, L. 2003, ApJ, 593, 56

Diemand, J., Kuhlen, M., Madau, P., et al. 2008, Nature, 454, 735
Dotti, M., Colpi, M., Pallini, S., Perego, A., \& Volonteri, M. 2013, ApJ, 762, 68

Dotti, M., Volonteri, M., Perego, A., et al. 2010, MNRAS, 402, 682

Dubois, Y., Devriendt, J., Slyz, A., \& Teyssier, R. 2012, MNRAS, 420, 2662

Dubois, Y., Gavazzi, R., Peirani, S., \& Silk, J. 2013, MNRAS, 433, 3297

Dubois, Y., Volonteri, M., \& Silk, J. 2014, MNRAS, 440, 1590

Eisenstein, D. J., \& Hu, W. 1999, ApJ, 511, 5

Enoki, M., Inoue, K. T., Nagashima, M., \& Sugiyama, N. 2004, ApJ, 615, 19

Fan, L., Lapi, A., De Zotti, G., \& Danese, L. 2008, ApJ, 689, L101

Ferrarese, L. 2002, ApJ, 578, 90

Ferrarese, L., \& Merritt, D. 2000, ApJ, 539, L9

Ferrarese, L., Côté, P., Dalla Bontà, E., et al. 2006, ApJ, 644, L21

Fitchett, M. J., \& Detweiler, S. 1984, MNRAS, 211, 933

Forte, J. C., Vega, E. I., \& Faifer, F. 2012, MNRAS, 421, 635

Gadotti, D. A. 2009, MNRAS, 393, 1531

Gaskell, C. M. 2009, ArXiv e-prints, arXiv:0908.0328

Gebhardt, K., \& Thomas, J. 2009, ApJ, 700, 1690

Gebhardt, K., Bender, R., Bower, G., et al. 2000, ApJ, 539, L13

Gilli, R., Comastri, A., \& Hasinger, G. 2007, A\&A, 463, 79

Gnedin, O. Y., Ostriker, J. P., \& Tremaine, S. 2014, ApJ, 785, 71

Granato, G. L., De Zotti, G., Silva, L., Bressan, A., \& Danese, L. 2004, ApJ, 600, 580

Granato, G. L., Silva, L., Lapi, A., et al. 2006, MNRAS, 368, L72

Greene, J. E., Peng, C. Y., \& Ludwig, R. R. 2010a, ApJ, 709, 937

Greene, J. E., Peng, C. Y., Kim, M., et al. 2010b, ApJ, 721, 26

Gültekin, K., Richstone, D. O., Gebhardt, K., et al. 2009, ApJ, 698, 198

Guo, Q., White, S., Angulo, R. E., et al. 2013, MNRAS, 428, 1351

Guo, Q., White, S., Li, C., \& Boylan-Kolchin, M. 2010, MNRAS, 404,1111

Haardt, F., \& Madau, P. 1996, ApJ, 461, 20

Haehnelt, M. G., \& Kauffmann, G. 2000, MNRAS, 318, L35

Häring, N., \& Rix, H.-W. 2004, ApJ, 604, L89

Hausman, M. A., \& Ostriker, J. P. 1978, ApJ, 224, 320

Heckman, T. M., Kauffmann, G., Brinchmann, J., et al. 2004, ApJ, 613, 109

Herrmann, F., Hinder, I., Shoemaker, D. M., Laguna, P., \& Matzner, R. A. 2007, Phys. Rev. D, 76, 084032

Hickox, R. C., Mullaney, J. R., Alexander, D. M., et al. 2014, ApJ, 782, 9

Hilz, M., Naab, T., Ostriker, J. P., et al. 2012, MNRAS, 425, 3119

Hirschmann, M., Khochfar, S., Burkert, A., et al. 2010, MNRAS, 407, 1016

Hopkins, P. F., Murray, N., \& Thompson, T. A. 2009, MNRAS, 398, 303

Hopkins, P. F., Richards, G. T., \& Hernquist, L. 2007, ApJ, 654, 731

Hopkins, P. F., Bundy, K., Croton, D., et al. 2010, ApJ, 715, 202

Islam, R. R., Taylor, J. E., \& Silk, J. 2004, MNRAS, 354, 443

Jaffe, A. H., \& Backer, D. C. 2003, ApJ, 583, 616

Joung, M. R., Cen, R., \& Bryan, G. L. 2009, ApJ, 692, L1

Kawakatu, N., \& Umemura, M. 2002, MNRAS, 329, 572

Kawakatu, N., Umemura, M., \& Mori, M. 2003, ApJ, 583, 85

Kocsis, B., \& Sesana, A. 2011, MNRAS, 411, 1467

Komatsu, E., Dunkley, J., Nolta, M. R., et al. 2009, ApJS, 180, 330 
Komossa, S., \& Merritt, D. 2008, ApJ, 683, L21

Koppitz, M., Pollney, D., Reisswig, C., et al. 2007, Physical Review Letters, 99, 041102

Kormendy, J., \& Bender, R. 2011, Nature, 469, 377

Kormendy, J., Bender, R., \& Cornell, M. E. 2011, Nature, 469, 374

Kormendy, J., \& Ho, L. C. 2013, ARA\&A, 51, 511

Kormendy, J., \& Richstone, D. 1995, ARA\&A, 33, 581

Lackner, C. N., Cen, R., Ostriker, J. P., \& Joung, M. R. 2012, MNRAS, 425, 641

Lang, P., Wuyts, S., Somerville, R. S., et al. 2014, ApJ, 788, 11

Lapi, A., Shankar, F., Mao, J., et al. 2006, ApJ, 650, 42

Läsker, R., Ferrarese, L., van de Ven, G., \& Shankar, F. 2014, ApJ, 780, 70

Leauthaud, A., George, M. R., Behroozi, P. S., et al. 2012, ApJ, 746,95

Li, S., Liu, F. K., Berczik, P., Chen, X., \& Spurzem, R. 2012, ApJ, 748, 65

Li, Y., Haiman, Z., \& Mac Low, M.-M. 2007, ApJ, 663, 61

Lin, Y.-T., Brodwin, M., Gonzalez, A. H., et al. 2013, ApJ, 771, 61

Lin, Y.-T., Ostriker, J. P., \& Miller, C. J. 2010, ApJ, 715, 1486

Lin, Y.-T., Stanford, S. A., Eisenhardt, P. R. M., et al. 2012, ApJ, 745, L3

Lippai, Z., Frei, Z., \& Haiman, Z. 2009, ApJ, 701, 360

Liu, F. K., \& Chen, X. 2013, ApJ, 767, 18

Lousto, C. O., Campanelli, M., Zlochower, Y., \& Nakano, H. 2010, Classical and Quantum Gravity, 27, 114006

Lousto, C. O., \& Zlochower, Y. 2008, Phys. Rev. D, 77, 044028

—. 2011, Physical Review Letters, 107, 231102

Lousto, C. O., Zlochower, Y., Dotti, M., \& Volonteri, M. 2012, Phys. Rev. D, 85, 084015

Madau, P., \& Quataert, E. 2004, ApJ, 606, L17

Maiolino, R., Gallerani, S., Neri, R., et al. 2012, MNRAS, 425, L66

Malbon, R. K., Baugh, C. M., Frenk, C. S., \& Lacey, C. G. 2007, MNRAS, 382, 1394

Maoz, E. 1993, MNRAS, 263, 75

Marconi, A., \& Hunt, L. K. 2003, ApJ, 589, L21

Marconi, A., Risaliti, G., Gilli, R., et al. 2004, MNRAS, 351, 169

McConnell, N. J., \& Ma, C.-P. 2013, ApJ, 764, 184

McLeod, K. K., \& Bechtold, J. 2009, ApJ, 704, 415

McLure, R. J., Pearce, H. J., Dunlop, J. S., et al. 2013, MNRAS, 428, 1088

McWilliams, S. T., Ostriker, J. P., \& Pretorius, F. 2012, ArXiv e-prints, arXiv:1211.4590

-. 2014, ApJ, 789, 156

Mendel, J. T., Simard, L., Palmer, M., Ellison, S. L., \& Patton, D. R. 2014, ApJS, 210, 3

Menou, K., \& Haiman, Z. 2004, ApJ, 615, 130

Merloni, A., \& Heinz, S. 2008, MNRAS, 388, 1011

Merloni, A., Rudnick, G., \& Di Matteo, T. 2004, MNRAS, 354, L37

Merloni, A., Bongiorno, A., Bolzonella, M., et al. 2010, ApJ, 708, 137

Milosavljević, M., \& Merritt, D. 2003, in American Institute of Physics Conference Series, Vol. 686, The Astrophysics of Gravitational Wave Sources, ed. J. M. Centrella, 201-210

Moster, B. P., Naab, T., \& White, S. D. M. 2013, MNRAS, 428, 3121

Natarajan, P. 2004, in Astrophysics and Space Science Library, Vol. 308, Supermassive Black Holes in the Distant Universe, ed. A. J. Barger, 127

Natarajan, P. 2011, ArXiv e-prints, arXiv:1105.4902

Natarajan, P., \& Treister, E. 2009, MNRAS, 393, 838

Navarro, J. F., Frenk, C. S., \& White, S. D. M. 1997, ApJ, 490, 493

Netzer, H. 2009, MNRAS, 399, 1907

Nipoti, C., Treu, T., Auger, M. W., \& Bolton, A. S. 2009, ApJ, 706, L86

Oser, L., Naab, T., Ostriker, J. P., \& Johansson, P. H. 2012, ApJ, 744,63
Oser, L., Ostriker, J. P., Naab, T., Johansson, P. H., \& Burkert, A. 2010, ApJ, 725, 2312

O'Shea, B. W., Bryan, G., Bordner, J., et al. 2004, ArXiv Astrophysics e-prints, arXiv:astro-ph/0403044

Ostriker, J. P., Choi, E., Ciotti, L., Novak, G. S., \& Proga, D. 2010, ApJ, 722, 642

Peres, A. 1962, Physical Review, 128, 2471

Pratt, G. W., Croston, J. H., Arnaud, M., \& Böhringer, H. 2009, A\&A, 498, 361

Rajagopal, M., \& Romani, R. W. 1995, ApJ, 446, 543

Rashkov, V., \& Madau, P. 2014, ApJ, 780, 187

Ravi, V., Wyithe, J. S. B., Hobbs, G., et al. 2012, ApJ, 761, 84

Rosario, D. J., Santini, P., Lutz, D., et al. 2012, A\&A, 545, A45

Salvaterra, R., Haardt, F., \& Volonteri, M. 2007, MNRAS, 374, 761

Salviander, S., \& Shields, G. A. 2013, ApJ, 764, 80

Sani, E., Marconi, A., Hunt, L. K., \& Risaliti, G. 2011, MNRAS, 413, 1479

Schnittman, J. D., \& Buonanno, A. 2007, ApJ, 662, L63

Schulze, A., \& Wisotzki, L. 2014, MNRAS, 438, 3422

Sesana, A. 2012, Advances in Astronomy, 2012, arXiv:1110.6445

-. 2013a, Classical and Quantum Gravity, 30, 224014

-. 2013b, MNRAS, 433, L1

Sesana, A., Haardt, F., Madau, P., \& Volonteri, M. 2004, ApJ, 611,623

Sesana, A., Vecchio, A., \& Volonteri, M. 2009, MNRAS, 394, 2255

Shankar, F. 2013, Classical and Quantum Gravity, 30, 244001

Shankar, F., Bernardi, M., \& Haiman, Z. 2009a, ApJ, 694, 867

Shankar, F., Weinberg, D. H., \& Miralda-Escudé, J. 2009b, ApJ, 690,20

- 2013, MNRAS, 428, 421

Shen, Y. 2009, ApJ, 704, 89

Shields, G. A., Gebhardt, K., Salviander, S., et al. 2003, ApJ, 583,124

Sijacki, D., Springel, V., \& Haehnelt, M. G. 2009, MNRAS, 400, 100

Soltan, A. 1982, MNRAS, 200, 115

Somerville, R. S., Gilmore, R. C., Primack, J. R., \& Domínguez, A. 2012, MNRAS, 423, 1992

Springel, V., White, S. D. M., Jenkins, A., et al. 2005, Nature, 435,629

Treister, E., Schawinski, K., Volonteri, M., \& Natarajan, P. 2013, ApJ, 778, 130

Treister, E., Urry, C. M., \& Virani, S. 2009, ApJ, 696, 110

Tremaine, S., Gebhardt, K., Bender, R., et al. 2002, ApJ, 574, 740

Umemura, M. 2001, ApJ, 560, L29

van der Wel, A., Holden, B. P., Zirm, A. W., et al. 2008, ApJ, 688,48

van Dokkum, P. G., Franx, M., Kriek, M., et al. 2008, ApJ, 677, L5

van Haasteren, R., Levin, Y., Janssen, G. H., et al. 2011, MNRAS, 414, 3117

van Meter, J. R., Miller, M. C., Baker, J. G., Boggs, W. D., \& Kelly, B. J. 2010, ApJ, 719, 1427

Volonteri, M., \& Ciotti, L. 2013, ApJ, 768, 29

Volonteri, M., Madau, P., Quataert, E., \& Rees, M. J. 2005, ApJ, 620,69

Volonteri, M., Natarajan, P., \& Gültekin, K. 2011, ApJ, 737, 50

Volonteri, M., \& Perna, R. 2005, MNRAS, 358, 913

Volonteri, M., Salvaterra, R., \& Haardt, F. 2006, MNRAS, 373, 121

Volonteri, M., Sikora, M., Lasota, J.-P., \& Merloni, A. 2013, ApJ, 775,94

Woo, J.-H., Treu, T., Malkan, M. A., \& Blandford, R. D. 2008, ApJ, 681, 925

Wyithe, J. S. B., \& Loeb, A. 2003, ApJ, 590, 691

Yoo, J., Miralda-Escudé, J., Weinberg, D. H., Zheng, Z., \& Morgan, C. W. 2007, ApJ, 667, 813

Yu, Q., \& Tremaine, S. 2002, MNRAS, 335, 965

Zhang, X., Lu, Y., \& Yu, Q. 2012, ApJ, 761, 5 